\title{
Water wave diffraction by a cylinder array. Part 2. Irregular waves
}

\author{
By C. O. G. OHL†, P. H. TAYLOR, R. EATOCK TAYLOR \\ AND A. G. L. BOR THWICK
}

Department of Engineering Science, University of Oxford, Parks Road, Oxford OX1 3PJ, UK

(Received 15 June 2000 and in revised form 14 December 2000)

Diffraction of irregular waves, focused wave groups, and random seas by an array of vertical bottom-mounted circular cylinders is investigated using theoretical, computational and experimental methods. This is an extension of our study of such an array in regular waves, reported in Part 1. Linear focused wave group theory is reviewed as a method for predicting the probable shape of extreme events from random wave spectra. Measurements are presented of the free surface elevation distribution in the vicinity of a multi-column structure in an offshore basin when subjected to irregular waves having peak frequencies and significant wave heights in the range $0.449<k_{p} a<0.555$ and $0.114<H_{s}<0.124$ respectively, where $a$ is the cylinder radius. Analytical linear diffraction theory is extended for application to focused wave groups and random seas. Experimental irregular wave data are analysed for comparison with this theory. Linear diffraction theory for random seas is shown to give an excellent prediction of incident wave spectral diffraction, while linear diffraction theory for focused wave groups works well for linearized extreme events. Due to the phase shifting of incident wave spectral components, diffraction is shown to generate focused wave groups in the vicinity of the cylinder array.

\section{Introduction}

As discussed by Tromans, Anaturk \& Hagemeijer (1991), design criteria for offshore structures located in the North Sea and the Gulf of Mexico are largely driven by the estimated environmental loads induced by extreme storms. In this structural design and assessment, the random condition is conventionally modelled using a deterministic, monochromatic, periodic wave theory that assumes the wave height and period to correspond to some extreme environmental condition (such as the highest wave arising on average in a three hour interval in the sea state of the most extreme storm expected in a one hundred year interval). Alternatively, a computationally demanding time domain simulation, including the random, spectral, and directional properties of the extreme storm, can be run to determine structural response within a given time interval (such as three hours in the above case). However, to avoid time domain computations while retaining accuracy, Tromans et al. reformulated the deterministic method using a probabilistic, focused wave group (or NewWave) analysis. Accounting for the irregular shape and random background of large ocean waves, this method models the sea as a set of independent wavelets of random

$\dagger$ Present address: c/o Human Resources, Costain Limited, Costain House, Nicholson's Walk, Maidenhead, Berkshire SL6 1LN, UK. 
amplitude. Focused wave groups then occur at a point where the crests of many of the wavelets coincide. By calculating the most probable ocean surface elevation around a crest, this NewWave analysis includes much of the spectral properties of the sea state. Note, however, that the basic statistical theory concerning the shapes of extremes in a Gaussian random process was first developed by Lindgren (1980).

Jonathan \& Taylor (1995) provided detailed comparisons between the predictions of focused wave group theory and measurements made in the North Sea. The nine hours of analysed data were from a storm with significant wave height and peak period that were relatively constant at $H_{s}=12 \mathrm{~m}$ and $T_{p}=14 \mathrm{~s}$ respectively, and a spectral tail decaying with $\omega^{-4}$. The large nonlinear crests from the record were linearized by subtracting the averaged large crest and large trough profiles. This effectively removes second-order nonlinearity, producing a near-linear free surface profile. Comparisons of the linearized free surface elevation time profiles to the predictions of focused wave group theory were subsequently made, and Jonathan \& Taylor concluded that 'for large crests, NewWave gives excellent agreement with offshore measurements.'

In a theoretical study of the diffraction of random seas, Eatock Taylor \& Sincock (1989) developed a hybrid element technique to model the hydrodynamics, leading to results for the modification of the mean-square surface amplitude spectrum caused by diffraction. Whereas the resultant profiles resembled those from regular wave calculations, the overall magnification effects were of course substantially reduced in multi-frequency waves. In addition, Eatock Taylor \& Wu (1997) computed theoretical results for the linear diffraction of focused waves from a single cylinder.

While much research into the computation of wave diffraction has been undertaken, the majority of this has concentrated on the development of linear and higher-order potential flow theories or fully nonlinear analyses for regular waves. However, it is generally accepted that regular waves are unrealistic for the simulation of extreme storm design conditions, for which focused wave group events or random wave spectra are more representative.

In light of the above, this paper investigates the diffraction of focused wave groups and random seas by an array of four vertical bottom-mounted circular cylinders using theoretical, computational, and experimental methods. A comprehensive discussion of the experimental setup, data acquisition, and analysis of regular wave results is given in Part 1 by Ohl et al. (2001). Free surface elevation is the defining aspect used to test the potential theory developed. The principal objectives are to expand diffraction theory for cylinder arrays to cover focused wave groups and random seas at first order, to analyse free surface elevation time histories collected from the irregular wave experiments, and to compare these results with those predicted by the first-order theory for incident focused wave groups as well as assessing the diffraction theories for focused wave groups and random seas developed herein.

\section{Linear diffraction theory for focused wave groups and random seas}

The focused wave group, also known as an extreme or freak wave, is composed of a full wave spectrum in which all components come into phase at a given point in the field. The following details the formulation of the equations necessary to define the incident wave prior to the diffraction computation. A general discussion of Linton \& Evans' (1990) linear diffraction theory for regular waves is given in Part 1. 


\subsection{Incident focused wave groups}

Tromans et al. (1991) outlined the theory of focused wave groups in random seas beginning from a representation of the ocean surface by a sum of numerous wavelets,

$$
\eta_{I}(x, y, t)=\sum_{n} A_{n} \cos \left(k_{n} x \cos \beta_{n}+k_{n} y \sin \beta_{n}-\omega_{n} t+\varsigma_{n}\right),
$$

where $\omega_{n}$ is the wave frequency, $k_{n}$ is the wavenumber, $\varsigma_{n}$ is a random phase angle and $\beta_{n}$ is the angle of incidence with the $x$-axis, all associated with the wave component $n$. From the one-sided directional surface power spectrum $f\left(\omega_{n}, \beta_{n}\right) S\left(\omega_{n}\right)$, the expected value of the amplitude $A_{n}$ is given by

$$
E\left(A_{n}\right)=\sqrt{2 S\left(\omega_{n}\right) f\left(\omega_{n}, \beta_{n}\right) \Delta \omega \Delta \beta},
$$

where $f$ is the directional component associated with the frequency and angle of incidence, $S$ is the magnitude of the surface power spectrum of component $n$, and $A_{n}$ is a Rayleigh distributed random variable. Assuming a uni-directional wave field, (2.2) becomes

$$
E\left(A_{n}\right)=\sqrt{2 S\left(\omega_{n}\right) \Delta \omega} .
$$

An extreme wave crest occurs when many of the frequency components come into phase. Tromans et al. (1991) discuss the average shape of an extreme event in a linear random Gaussian sea state. This mean shape has the appearance of a focused wave group

$$
\eta_{I}^{*}(X, \tau)=\frac{\alpha}{\sigma^{2}} \sum_{n} C_{n} \cos \left(k_{n} X-\omega_{n} \tau\right)
$$

where $\eta_{I}^{*}$ is the free surface profile of the wave group, $\sigma$ is the standard deviation, $C_{n}=\left(S\left(\omega_{n}\right) \Delta \omega_{n}\right), X=x-x_{0}, \tau=t-t_{0}$ and $\left(x_{0}, t_{0}\right)$ is the position and time of occurrence of a large crest. This average shape of a large crest of maximum elevation $\alpha$ is a model for extreme waves that is commonly referred to as NewWave.

From (2.4), a large crest is then generated when all of the wavelets comprising the group come into phase, producing a free surface elevation which is identical in shape on average to a scaled version of the autocorrelation function $\rho(x, \tau)$ for the wave spectrum.

\subsection{Diffracted focused wave groups}

Because complicated nonlinear interactions between waves at different frequencies are not accounted for, first-order diffraction theory (Linton \& Evans 1990) is simple to apply to the case of focused wave groups . This is accomplished by linear superposition of the diffracted wave field for each wavenumber component of the incident wave. From (2.4) above, the free surface elevation due to the scattering of an incident focused wave group (i.e. a diffracted extreme crest) can be calculated through

$$
\eta_{D}(x, y, \tau)=\frac{\alpha}{\sigma^{2}} \sum_{n} C_{n} \operatorname{Re}\left\{\phi_{n}(x, y) \mathrm{e}^{-\mathrm{i}\left(k_{n} x_{0}+\omega_{n} \tau\right)}\right\},
$$

where $\tau=t-t_{0}$ and $X=x-x_{0}$, with the focus occurring in the $y$-plane at $\left(x_{0}, y, t_{0}\right)$. The heading $\beta$ is assumed to be zero and the diffraction coefficient for the $n$th spectral component is $\phi_{n}$ (which may be obtained using the method of Linton \& Evans).

However, keeping the amplitude of each spectral component fixed, a random phase angle $\varsigma_{n}$ may be included in (2.5) above to calculate the free surface elevation for an 
imperfectly focused wave group. This is then

$$
\begin{aligned}
\eta_{D}(x, y, \tau) & =\frac{\alpha}{\sigma^{2}} \sum_{n} C_{n} \operatorname{Re}\left\{\phi_{n}(x, y) \mathrm{e}^{-\mathrm{i}\left(k_{n} x_{0}+\omega_{n} \tau-\varsigma_{n}\right)}\right\} \\
& =\frac{\alpha}{\sigma^{2}} \sum_{n} C_{n} \operatorname{Re}\left\{\phi_{n}(x, y) \mathrm{e}^{-\mathrm{i}\left(k_{n} x_{0}+\omega_{n} \tau\right)} \mathrm{e}^{\mathrm{i} \varsigma_{n}}\right\},
\end{aligned}
$$

such that $0 \leqslant \varsigma_{n} \leqslant 2 \pi$ and $\left|\mathrm{e}^{\mathrm{i}_{\varsigma_{n}}}\right|=1$.

There exists a value of this phase angle $\varsigma_{n}$ such that the diffraction coefficient $\phi_{n}(x, y)$ for each component reaches its maximum value $\left|\phi_{n}(x, y)\right|$ at the same position and time. This phase angle is given by $\varsigma_{n}$ where

$$
\mathrm{e}^{\mathrm{i} \varsigma_{n}}=\mathrm{e}^{\mathrm{i} k_{n} x} \frac{\left|\phi_{n}(x, y)\right|}{\phi_{n}(x, y)} .
$$

Applied to (2.6) above, this produces the phase-modified focused wave group

$$
\eta_{D}(x, y, \tau)=\frac{\alpha}{\sigma^{2}} \sum_{n} C_{n} \operatorname{Re}\left\{\left|\phi_{n}(x, y)\right| \mathrm{e}^{\mathrm{i}\left(k_{n} X-\omega_{n} \tau\right)}\right\} .
$$

If the point of computation is then assumed to be the focus point, with $x=x_{0}$ and $t=t_{0}$ such that $X=0$ and $\tau=0,(2.8)$ may be written as

$$
\eta_{M}(x, y)=\frac{\alpha}{\sigma^{2}} \sum_{n} C_{n}\left|\phi_{n}(x, y)\right| .
$$

This expression allows the computation of the maximum possible free surface elevation at a given point $(x, y)$ for an incident focused wave group (of fixed amplitude components but arbitrary phasing) with linear diffraction. To the authors' knowledge, (2.9) is novel.

The above method for computing a diffracted focused wave group is demonstrated in figures 1 to 10. As in the above cases of Linton \& Evans (1990), the figures encompass various geometric configurations while maintaining $a / h=0.5$, where $a$ is the cylinder radius and $2 h$ is the spacing (i.e. distance between cylinder centres for multiple cylinder cases). The majority of the figures contain three plots showing results for sections parallel to the $x$-axis as annotated (e.g. $y=0, h$, and $2 h$ in figure 2). However, indicating the cylinder positions for identical geometry cases, plan views of the contours of $\eta_{m}$ are interspersed within these sectioned figures. To aid in the discussion of results for the arrays of four cylinders, the two arrangements are designated by two separate headings $\beta$. The first configuration (with two cylinders upstream and two downstream) is referred to as heading $0^{\circ}$; and the second configuration (with one cylinder upstream, two abeam the centre and one downstream) is designated as heading $45^{\circ}$. (Note that use of this terminology is continued in subsequent discussion of experimental results.)

Incident focused wave groups are based on JONSWAP spectra with 120 wavenumber components and $\Delta k=k_{p} / 20$, where $k_{p}$ is the peak wavenumber of the incident wave spectrum. The wave spectra have been scaled such that the maximum free surface elevation for the undisturbed extreme crest from (2.4) is unity (i.e. $\eta_{I}^{*}\left(x_{0}, t_{0}\right)=1$ ).

The solid lines and contour lines in figures 1 to 10 indicate the maximum free surface elevation $\eta_{m}$ as computed through (2.9). Thus, a value of 2 indicates a maximum free surface elevation of twice the incident focused wave group, while 0.5 indicates half the incident elevation. Dotted lines represent the diffracted free surface elevation $\eta_{D}(x)$ at 


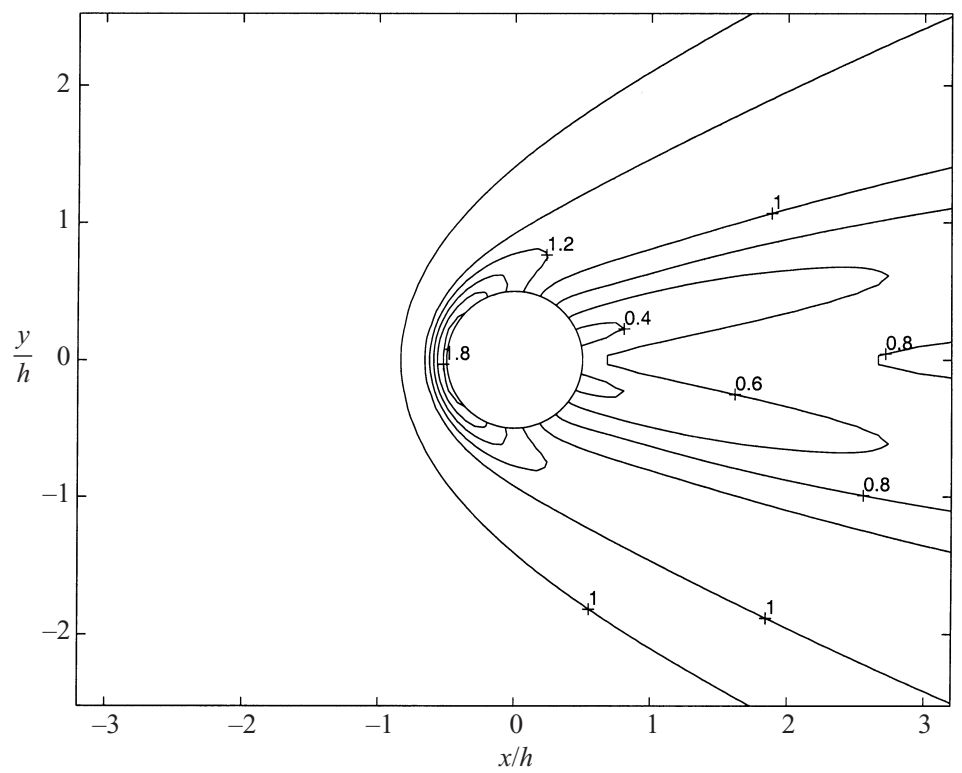

FIGURE 1. Contours of maximum free surface elevation; $N=1, \beta=0^{\circ}, k_{p} h=2 \pi, a / h=0.5$.

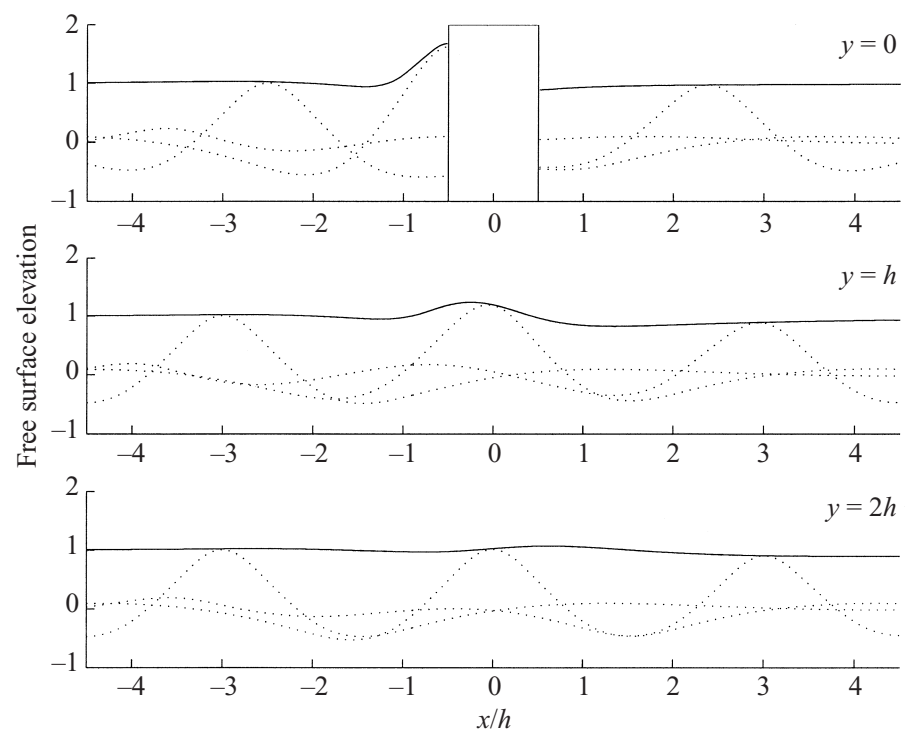

FIGURE 2. Maximum free surface elevation; $N=1, \beta=0^{\circ}, k_{p} h=\pi / 2, a / h=0.5$.

the instant of focus for an extreme wave crest (a NewWave event) focused at points representing either integer or half-integer values of $x / h$ as computed through (2.5).

Figures 1 to 3 provide results for a single cylinder $(N=1)$ centred at the origin. Figure 1 is a contour plot in plan view for the same incident wavenumber as figure 3 $\left(k_{p} h=2 \pi\right.$ corresponding to $\left.h / \lambda_{p}=1\right)$. Figure 1 shows the corresponding contour plot for the case $k_{p} h=\pi / 2$. Although having no physical meaning for the single cylinder case, $h$ has been set at $h=2 a$ to allow direct comparison with the multiple cylinder cases which follow. For this relatively simple single cylinder case, the diffracted extreme 


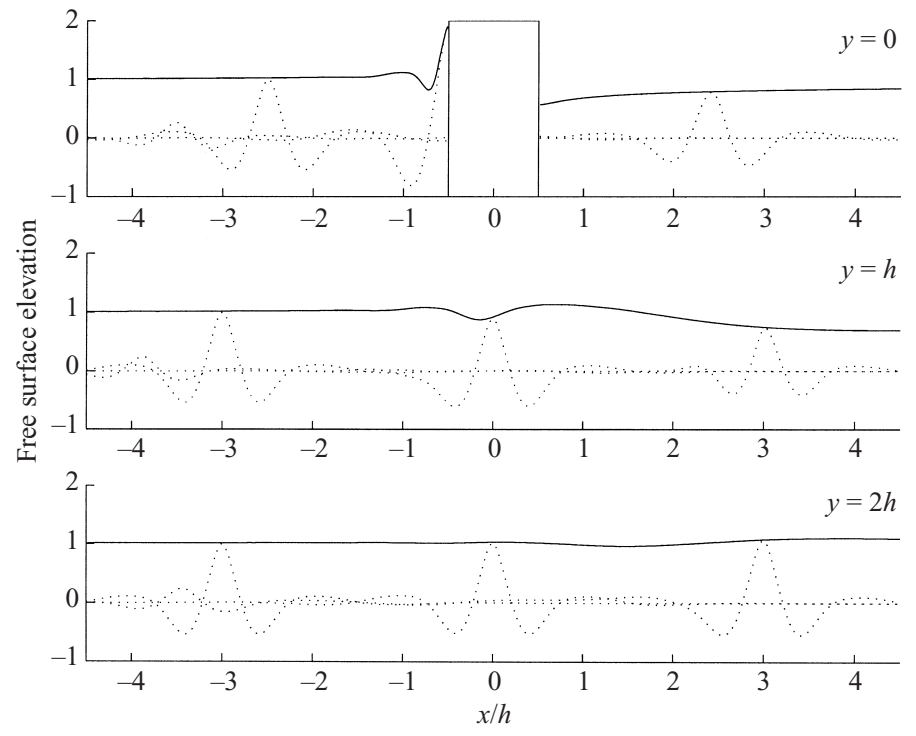

Figure 3. Maximum free surface elevation; $N=1, \beta=0^{\circ}, k_{p} h=2 \pi, a / h=0.5$.

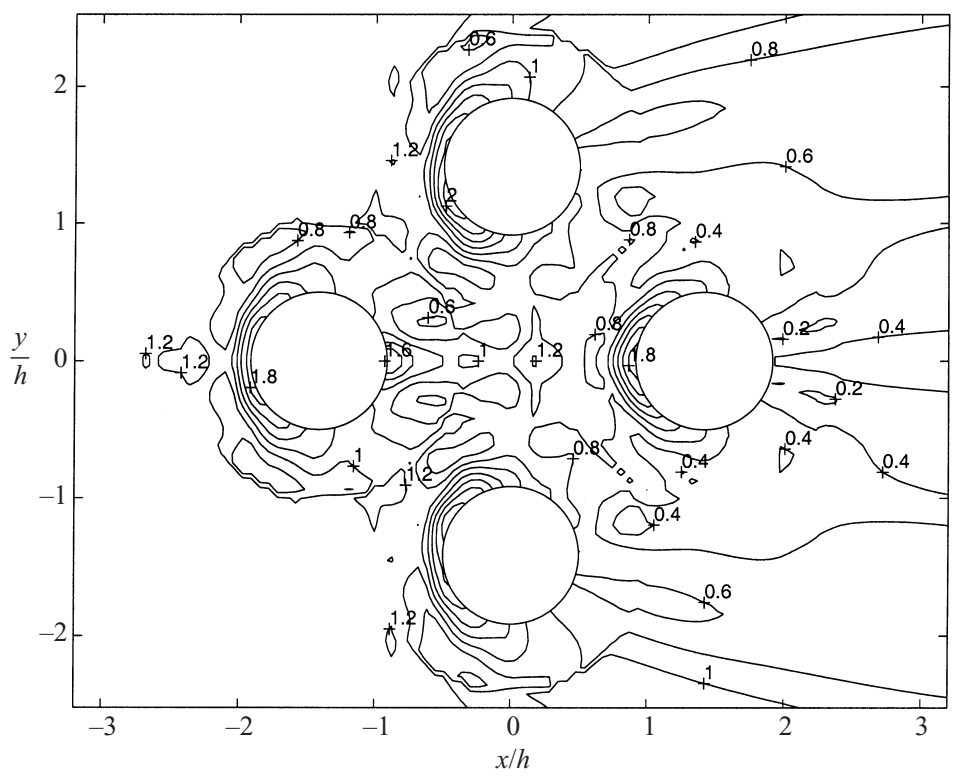

FIgURE 4. Contours of maximum free surface elevation; $N=4, \beta=45^{\circ}, k_{p} h=2 \pi, a / h=0.5$.

crests from (2.5) do achieve the maximum free surface elevation from (2.9) (i.e. the dotted lines are, in general, tangent to the solid line). However, as the geometry becomes more complex, interaction effects between cylinders becomes critical, and diffraction phase shifting defocuses incident extreme waves.

With heading $\beta=45^{\circ}$, figures 4 to 6 relate to a case with four cylinders $(N=4)$ centred at the corners of a square, side length $2 h$ and rotated through $\pi / 4$ radians (i.e. cylinders centred at $(-\sqrt{2} h, 0),(0, \sqrt{2} h),(\sqrt{2} h, 0)$ and $(0,-\sqrt{2} h))$. Again, the contour plot of figure 4 shows the geometry in plan view for the same case as figure 6 . 


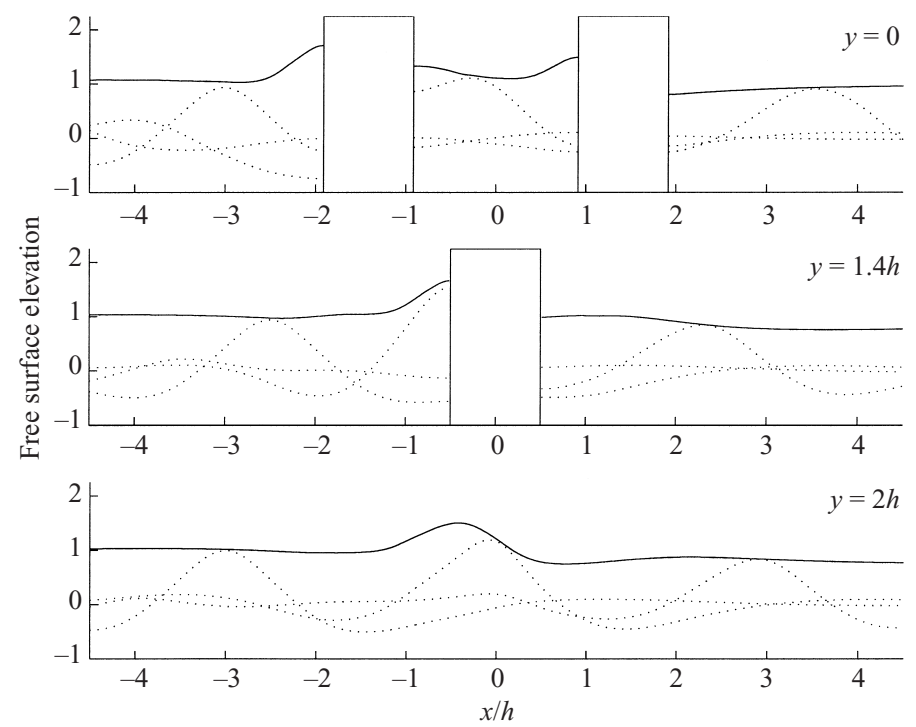

Figure 5. Maximum free surface elevation; $N=4, \beta=45^{\circ}, k_{p} h=\pi / 2, a / h=0.5$.

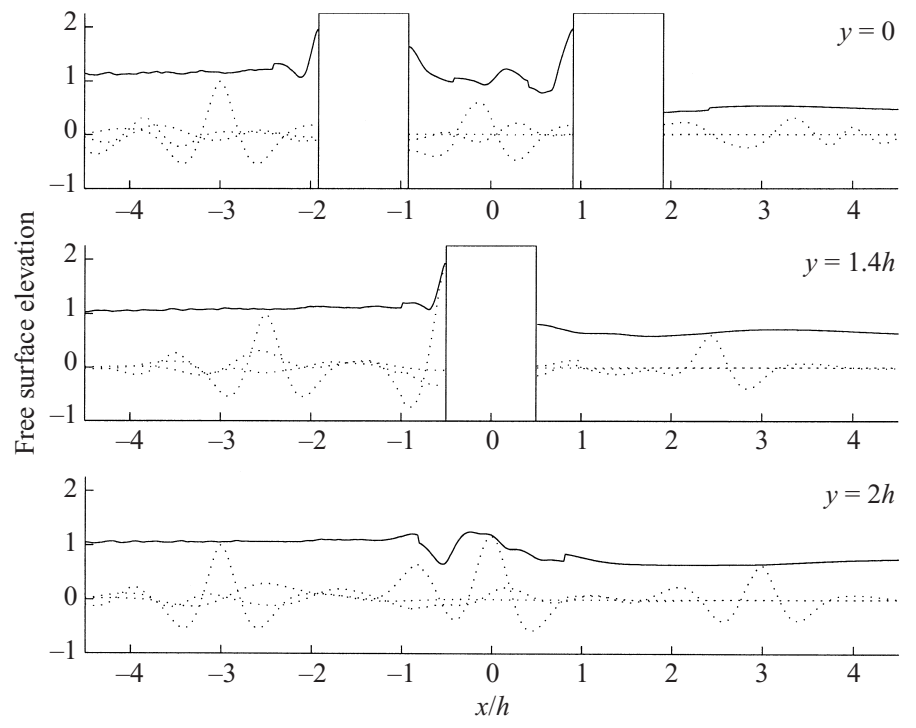

FIGURE 6. Maximum free surface elevation; $N=4, \beta=45^{\circ}, k_{p} h=2 \pi, a / h=0.5$.

Here a discrepancy between diffracted extreme crests from (2.5) and maximum free surface elevation from (2.9) becomes apparent, particularly for the shortest wavelength (highest wavenumber) plot of figure 6 with $k_{p} h=2 \pi$. This difference is most clear in the upper plots along the centreline and between the cylinders $(y=0, x / h=0)$, where the incident extreme wave crest has been shifted upstream a distance of approximately $-0.1 \mathrm{~h}$ and achieves an elevation that is some $50 \%$ of the maximum $\eta_{m}$ at that point.

Figures 7, 8 and 9 present a square configuration (i.e. $\beta=0$ ), with two cylinders upwave and two downwave of the origin. With a plan view of the configuration, figure 7 displays the contour plot corresponding to figures 8 and 9. Discrepancies 


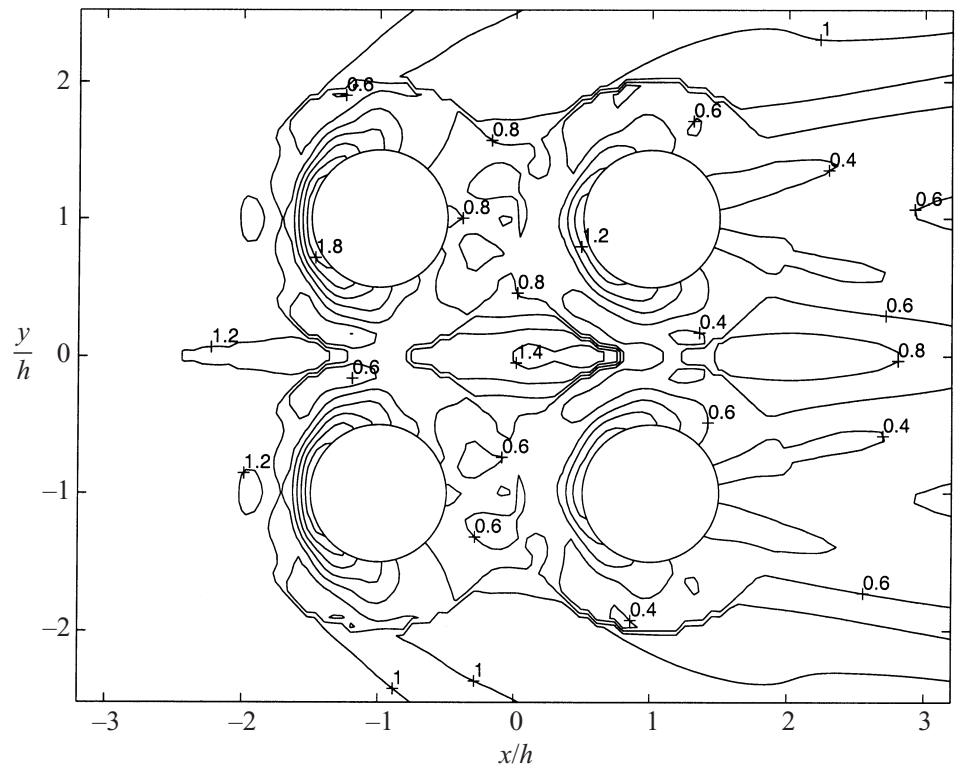

Figure 7. Contours of maximum free surface elevation; $N=4, \beta=0^{\circ}, k_{p} h=2 \pi, a / h=0.5$.

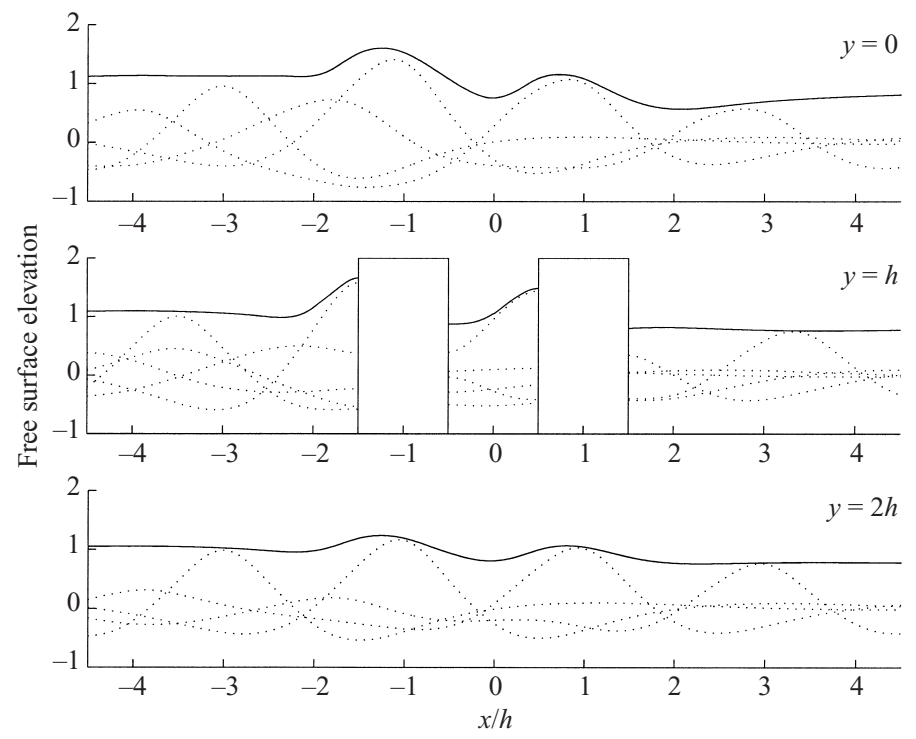

Figure 8. Maximum free surface elevation; $N=4, \beta=0^{\circ}, k_{p} h=\pi / 2, a / h=0.5$.

between diffracted extreme crests from (2.5) and maximum free surface elevations from (2.9) are most apparent in these figures. The largest differences are seen in the upper plots, for the centreline section along $y=0$, and these differences are perhaps most apparent for the highest wavenumber case of figure 9 with $k_{p} h=2 \pi$.

Finally, plotted along the plane $y=0$ for the same case as figure 9 , the dashed lines in the three plots of figure 10 show the wave groups achieving the maximum free surface elevation. These dashed lines are the free surface elevation $\eta$ for a phasemodified focused wave group from (2.6) with phase specified through (2.7) to achieve 


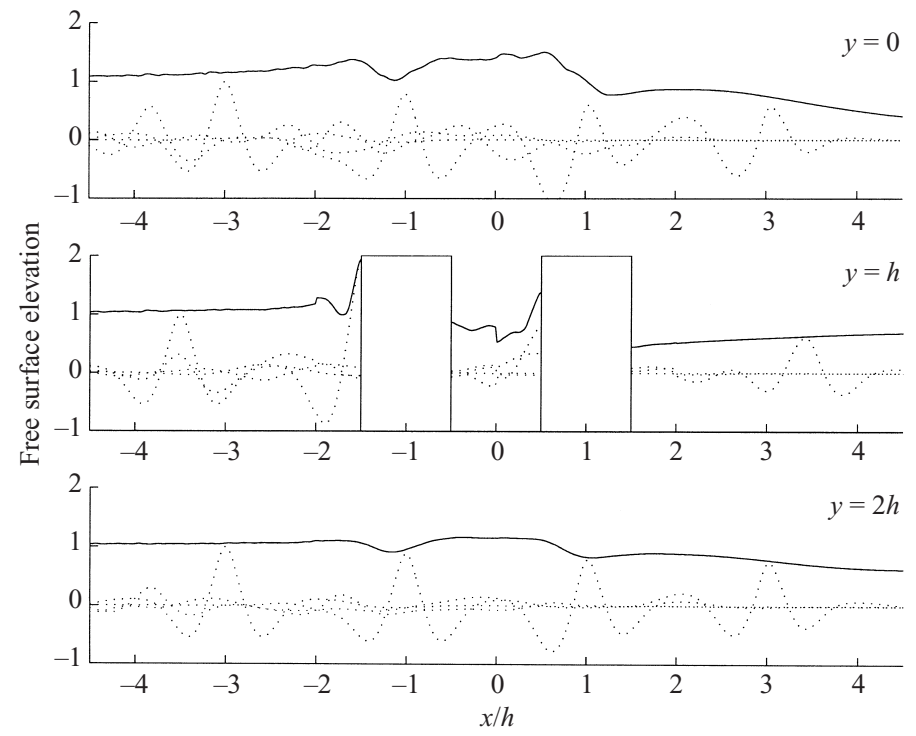

FIGURE 9. Maximum free surface elevation; $N=4, \beta=0^{\circ}, k_{p} h=2 \pi, a / h=0.5$.

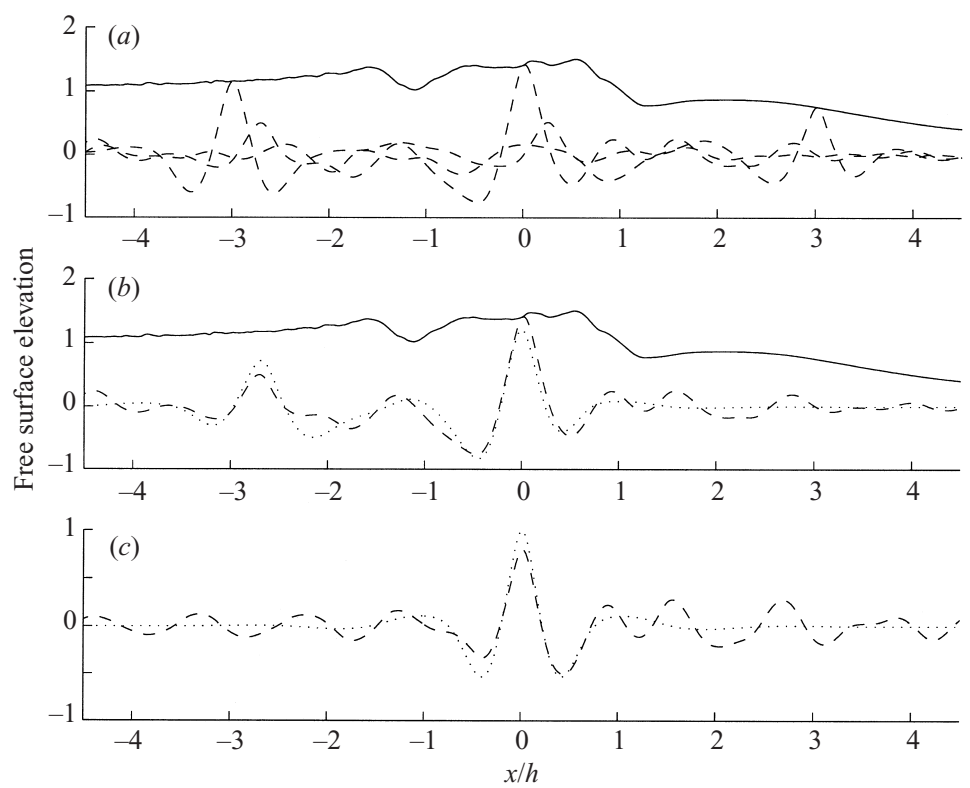

FIGURE 10. Maximum free surface elevation and varying incident wave forms in the plane $y=0$; $N=4, \beta=0^{\circ}, k_{p} h=2 \pi, a / h=0.5$.

the maximum free surface elevation of (2.9). Figure $10(a)$ is directly comparable to the upper plot of figure 9, with wave groups focused to obtain maximum value at all integer values of $x / h$. It is clear that the phase-modified wave groups of figure 10 obtain the maximum free surface elevation, while the unmodified groups of figure 9 do not. Figure 10(b) compares one such phase-modified diffracted wave group (dashed line from (2.6)) with the unmodified diffracted wave group (dotted line from (2.5)) focused at $x / h=0$. Again, it is clear that the dashed line, extending above the 


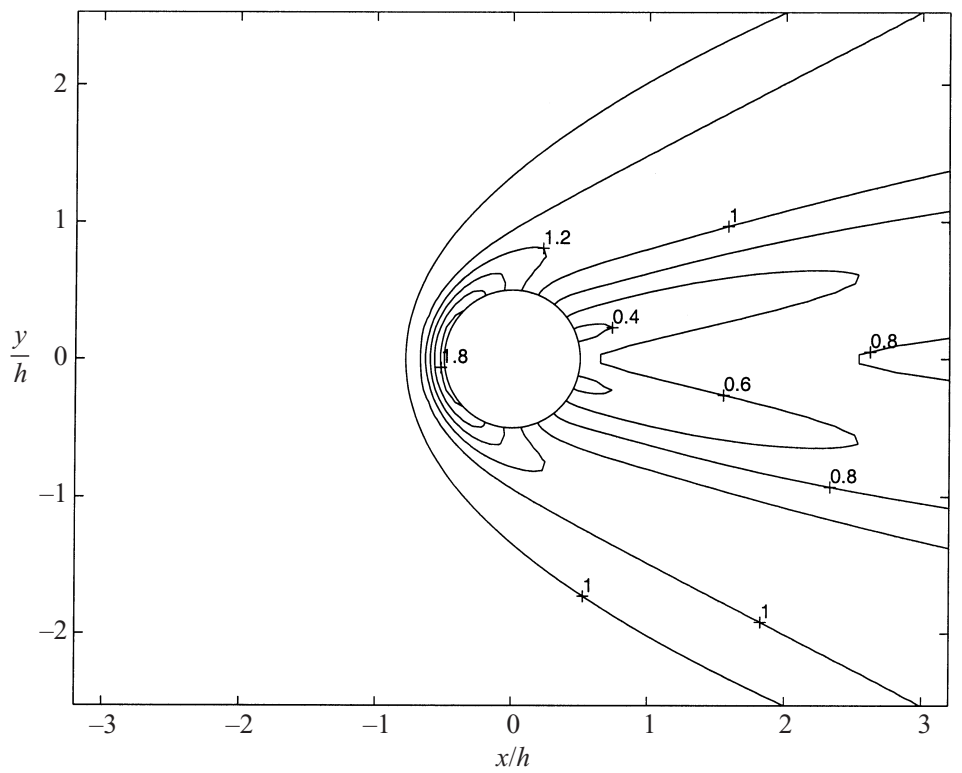

FIGURE 11. Diffracted modification to incident wave spectrum; $N=1, \beta=0^{\circ}, k_{p} h=2 \pi, a / h=0.5$.

dotted line to reach the maximum free surface elevation, is tangent to the solid line. Figure 10(c) compares the undisturbed incident wave forms for these phase-modified and unmodified cases. It is clear that the dotted line of the unmodified focused wave group from (2.4) is symmetric about its maximum, while the phase-modified dashed line represents a more complex incident wave form with lower undisturbed peak value.

\subsection{Random wave statistics}

The diffraction of a focused wave group has been treated above as a deterministic event, in which the diffraction of the group is computed through the sum of diffracted components of fixed amplitude. However, returning to the extreme value statistics of random seas, the maximum expected free surface elevation may be characterized in terms of the energy in the spectrum and the number of observed waves. Assuming that all large observed events conform to a Rayleigh distribution (see Dean \& Dalrymple 1991 for a complete derivation), the maximum expected incident free surface elevation $\eta_{i}$ is

$$
\eta_{i}=\sqrt{2 \ln (N) \sum_{n} S\left(\omega_{n}\right) \Delta \omega}
$$

where $N$ is the number of observed waves.

If the effects of diffraction due to the presence of a large structure are taken into account, the maximum expected diffracted free surface elevation $\eta_{d}$ at a point $(x, y)$ is then

$$
\eta_{d}(x, y)=\sqrt{2 \ln (N) \sum_{n}\left|\phi_{n}(x, y)\right|^{2} S\left(\omega_{n}\right) \Delta \omega} .
$$

It should be noted that the weighting of the diffraction coefficient $\phi_{n}$ is squared in this instance as it now modifies the energy density spectrum $S$ (in units of $\mathrm{m}^{2} \mathrm{~s}$ ), which 


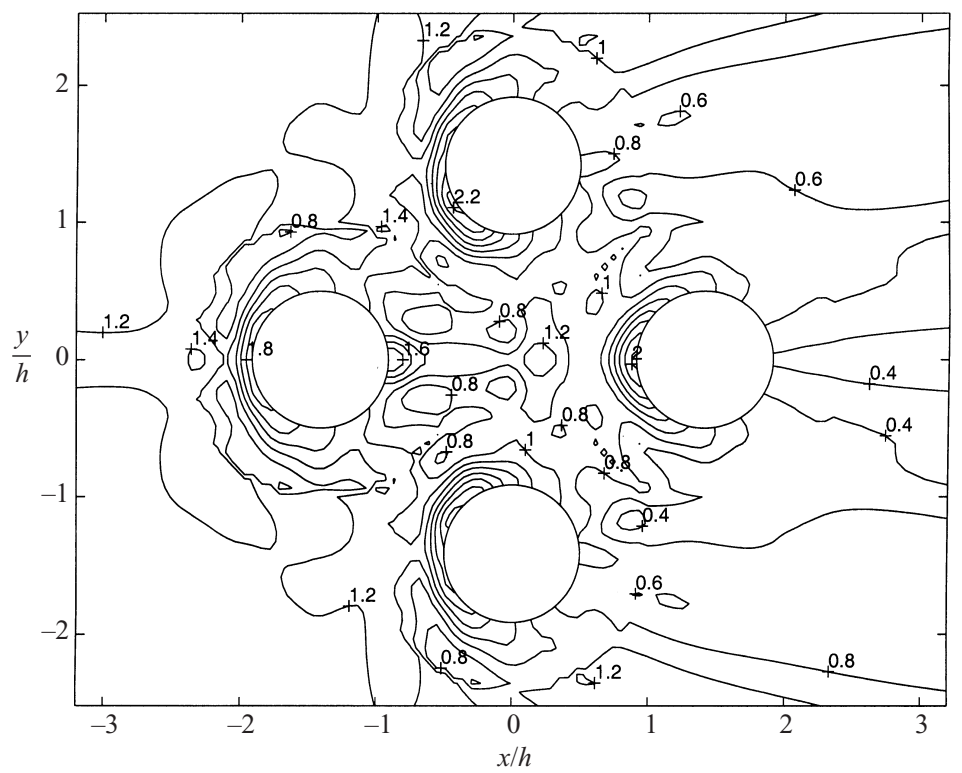

Figure 12. Diffracted modification to incident wave spectrum; $N=4, \beta=45^{\circ}, k_{p} h=2 \pi, a / h=0.5$.

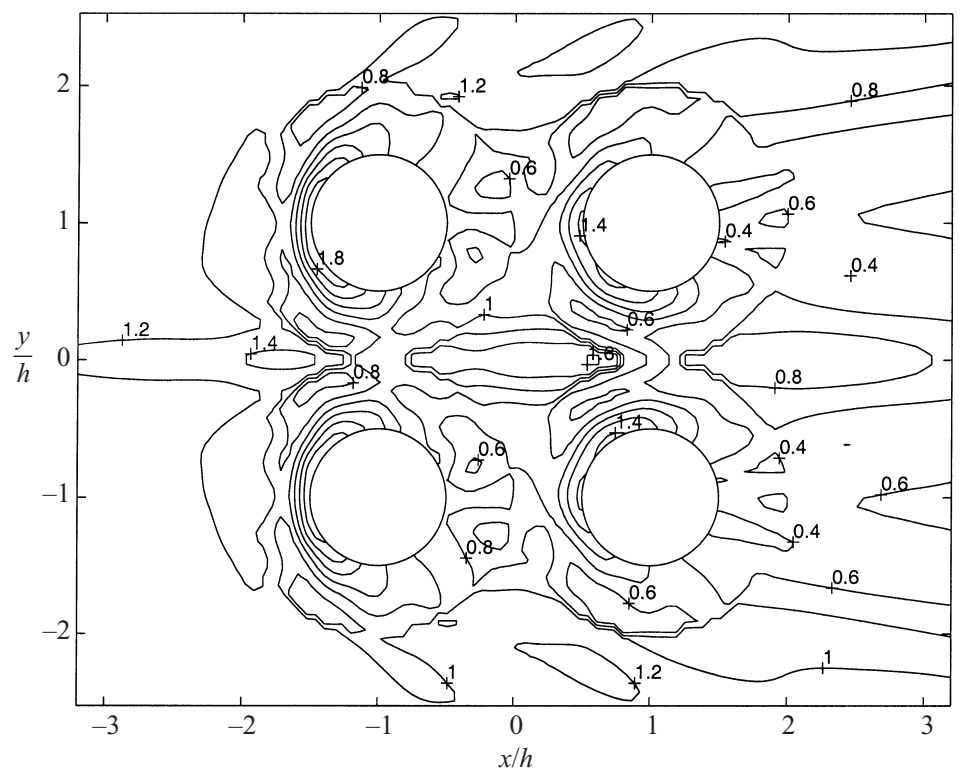

FIGURE 13. Diffracted modification to incident wave spectrum; $N=4, \beta=0^{\circ}, k_{p} h=2 \pi, a / h=0.5$.

has a squared relationship with the amplitude spectrum. This squared diffraction coefficient is consistent with the work of Eatock Taylor \& Sincock (1989), who examined the mean-square free surface elevation for random seas in the presence of a tension-leg platform structure.

The effect of this spectral diffraction relative to the maximum undisturbed free 
surface elevation may then be computed through

$$
\frac{\eta_{d}(x, y)}{\eta_{i}}=\sqrt{\frac{\sum_{n}\left|\phi_{n}(x, y)\right|^{2} S\left(\omega_{n}\right) \Delta \omega}{\sum_{n} S\left(\omega_{n}\right) \Delta \omega}} .
$$

An identical result may be obtained through the comparison of mean-square free surface elevation. This implies that (2.12) also represents the diffracted modification to such random sea characteristics as root-mean-square free surface elevation and significant wave height, as calculated based on the incident wave spectrum.

In addition, through this computation of the effects of diffraction on an incident spectrum, the average time history of the largest diffracted wave measured at a point in the vicinity of a structure may be computed from the diffracted spectrum as

$$
\eta_{d m}(x, y, \tau)=\frac{\alpha}{\sigma^{2}} \sum_{n} C_{n} \operatorname{Re}\left\{\left|\phi_{n}(x, y)\right|^{2} \mathrm{e}^{-\mathrm{i}\left(k_{n} x_{0}+\omega_{n} \tau\right)}\right\} .
$$

This differs from the above discussion of the diffraction of incident focused wave groups in that the amplitude components of the spectrum are now assumed to be random and modified by the presence of the structure.

Figures 11, 12 and 13 demonstrate the computation of (2.12) for cases identical to those of figures 1, 4 and 7 respectively. Again, the incident wave spectra are JONSWAP spectra of 120 wavenumber components with $\Delta k=k_{p} / 20$.

The former contour plots of maximum free surface elevation from (2.9), and the spectral diffraction modification plots from (2.12) are qualitatively very similar. However, even for the relatively simple single cylinder case of figure 11, there are some slight differences (note, for example, the somewhat larger contour at $\eta_{d} / \eta_{i}=0.4$ downwave of the cylinder in figure 1 as compared to figure 11).

For the more complex multiple cylinder geometries, differences between the two sets of figures are more notable, with diffracted spectral modification plots showing considerably larger and higher amplification regions. In figure 12 the contours upwave of the cylinders at $\eta_{d} / \eta_{i}=1.2$ extend over a larger region than those of figure 4; and the runup on the centreline and downwave cylinders in figure 12 reaches $\eta_{d} / \eta_{i}=2.2$ and 2 respectively, while the corresponding locations in figure 4 experience $\eta_{d} / \eta_{i}=2$ and 1.8. Similarly, figure 13 displays upwelling along the centreline $(y=0)$ and runup at the downwave cylinders approximately $20 \%$ above that of figure 7 (i.e. $\eta_{d} / \eta_{i}=1.4$ as compared to $\eta_{m p}=1.2$ ).

The relative increased effects of diffraction shown in figures 11,12 and 13 in comparison with figures 1,4 and 7 are due to inherent differences in the definitions of the waves. As (2.9) assumes the presence of an extreme focused wave group event, it treats the incident wave as a composition of several frequency components with fixed amplitude as dictated by focused wave group theory. This incident wave represents one extreme event from the undisturbed incident spectrum, and the diffraction of its wavenumber components is treated individually. In contrast, (2.12) computes the effect of diffraction on the incident wave spectrum and effectively produces a new random field at each point in the vicinity of a structure. This has the effect of modifying the underlying spectral statistics and any wave elevations computed from the spectrum. Thus, statistical elevations computed from the diffracted spectrum would be expected to show more amplification than corresponding diffracted focused wave groups. Ohl (1999) provides a more thorough treatment of this. 


\section{Results of irregular wave diffraction experiments}

Multi-cylinder diffraction experiments for regular and irregular waves were conducted at HR Wallingford. While the regular wave tests are discussed in Part 1, complete results for both are given by Ohl (1999). The analysis of free surface elevation data collected is described here in detail, as well as comparisons with predictions from analytical linear diffraction theories for random seas and focused wave groups developed above. Results of irregular wave tests in the empty wave basin have been compared to linear theory for focused wave groups, while tests with the model present are compared with linear wave diffraction theory for random seas and focused wave groups.

To facilitate comparisons between theory and experiment, a range of subscripts is used throughout this section: upper-case subscripts apply exclusively to focused wave group computations, whereas lower-case subscripts apply to all other quantities, such as spectral characteristics, mean-square free surface elevation, etc. Thus, incident waves will be distinguished through $I$ and $i$, while diffracted waves will be designated by $D$ and $d$. Focused wave group crests and troughs will be distinguished through $C$ and $T$ respectively. For theoretically computed quantities, the subscript $t$ will be used for mean-square free surface elevation, while the superscript $T$ will be used for focused wave group computations (this avoids any confusion with the trough subscript $T$ ).

\subsection{Data manipulation}

Measurements of the time variation of free surface elevation $\eta(t)$ were made at prescribed locations in the vicinity of the multi-cylinder model. Part 1 contains a detailed description of the exact model configuration, wave probe location, wave tank and testing procedures. In the case of the irregular wave tests, data were analysed for comparison with linear diffraction theories for random seas and focused wave groups, as presented above.

\subsubsection{Repeatability verification}

Incident waves for the irregular wave tests were produced by the wave basin paddles through a computer controlled process which extablished the spectral shape, peak frequency and significant wave height.

The repeatability of the tests is demonstrated in figure 14, which plots measurements of free surface elevation $\eta$ as a function of time $t$ at the reference wave probes for one of the irregular wave tests. For a representative section of data, the plots show reference wave probe time histories from the tests with the wave basin empty and from model tests at headings $0^{\circ}$ and $45^{\circ}$. Only a small portion of the test from the middle section of the data is displayed so that the signals may be readily compared. While slight discrepancies may be noted, the various lines are near to overlapping, and the individual tests may be taken as identical.

\subsubsection{Data record truncation}

The initial data records from the irregular wave tests encompassed measurements of free surface elevation $\eta(t)$ at $2^{14}(16384)$ data points covering a time of approximately $2^{9}$ (512) peak wave periods (i.e. sampling interval of $\Delta t=2^{-5} T_{p}$, where $T_{p}$ is the peak wave period). The highest frequency information that can be resolved through fast Fourier transforms (FFTs) is then the Nyquist critical frequency given by

$$
f_{c} \equiv \frac{1}{2 \Delta t}=\frac{1}{2^{-4} T_{p}}=16 f_{p} .
$$




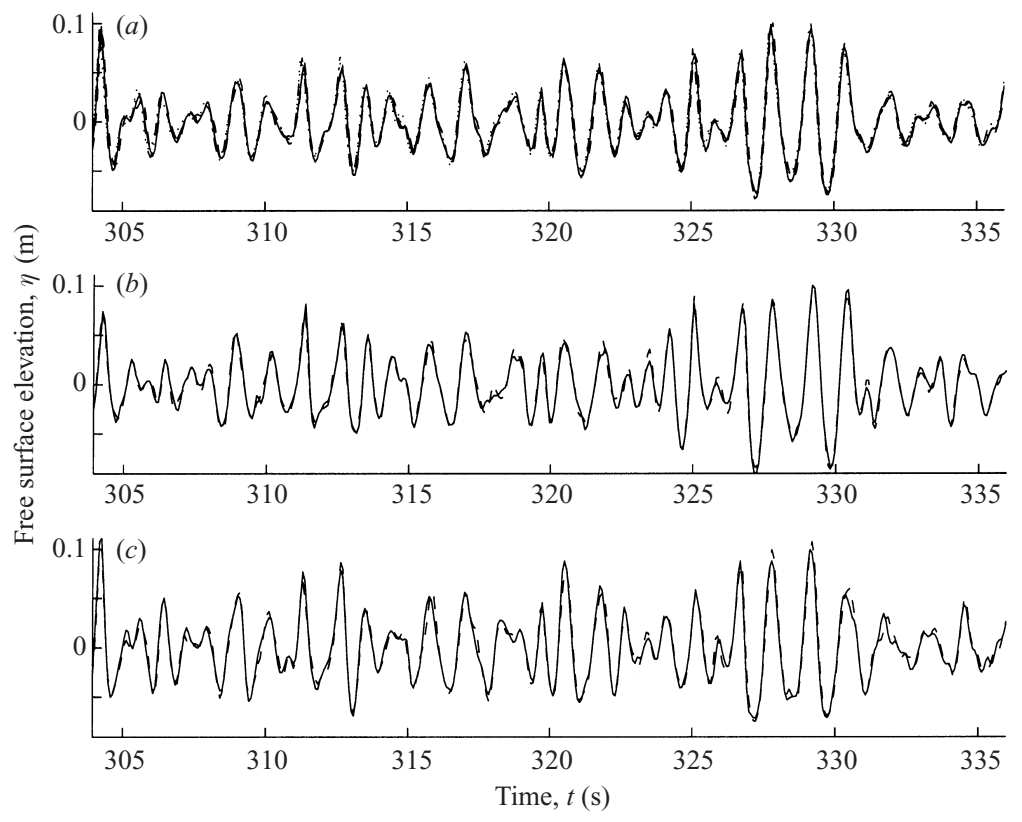

FiguRE 14. Test repeatability demonstrated through free surface elevation vs. time at reference wave probes: (a) empty tank, (b) $\beta=0^{\circ},(c) \beta=45^{\circ},\left(f_{p}=0.822499 \mathrm{~Hz}, H_{s}=0.11407 \mathrm{~m}\right)$.

To facilitate frequency analysis through FFTs, the original data record was truncated by removing every other data point.

To develop a sensibly sized bin for the frequency analysis, the truncated record produced above was then divided into four equal sections of length $2^{11}(2048)$ points. Each section was further divided into four equal subsections of length $2^{9}(512)$ points, producing one-sided spectra of $2^{8}(256)$ components with frequency discretisation of

$$
\Delta f=\frac{1}{2^{9} \Delta t}=\frac{1}{32} f_{p} .
$$

\subsubsection{Frequency analysis}

For the empty tank tests, FFTs of the truncated data sets were used to produce a frequency domain analysis for each subsection of $2^{9}(512)$ points and each wave probe location. Subsection spectra were then averaged to provide mean spectra for each section division of $2^{11}$ (2048) points. This provided 52 total wave spectra for each of the four sections (13 wave probe locations for each of four wave probe configurations). Representing measurements from points in close proximity, the 52 spectra were further averaged to produce one mean spectrum for each section.

Again, the resulting four section spectra were reasonably well correlated, and a mean wave spectrum was computed for each incident wave test. The three mean spectra produced in this manner (for tests 1,2 and 3 as shown in table 1) were used in subsequent calculations as the incident wave spectra at all positions for these three irregular wave conditions. It should be noted that no smoothing or running averaging has been used at any stage of the analysis.

From these incident wave spectra, the peak frequency $f_{p}$ has been identified as the frequency containing maximum energy, while significant wave height has been 


\begin{tabular}{|c|c|c|c|c|c|}
\hline Test & $\begin{array}{c}f_{p} \\
\text { selected } \\
{[\mathrm{Hz}]}\end{array}$ & $\begin{array}{c}H_{s} \\
\text { selected } \\
{[\mathrm{m}]}\end{array}$ & $\begin{array}{c}f_{p} \\
\text { computed } \\
{[\mathrm{Hz}]}\end{array}$ & $\underset{\substack{H_{s} \\
\text { computed }}}{\text { [m] }}$ & $\begin{array}{c}H_{s f i l t e r e d} \\
S\left(f>1.5 f_{p}\right) \\
{[\mathrm{m}]}\end{array}$ \\
\hline 1 & 0.8000 & 0.1300 & 0.8250 & 0.1141 & 0.1090 \\
\hline 2 & 0.7686 & 0.1400 & 0.7206 & 0.1208 & 0.1124 \\
\hline 3 & 0.7406 & 0.1400 & 0.7406 & 0.1239 & 0.1174 \\
\hline
\end{tabular}

computed from the spectra as

$$
H_{s}=4 \sigma=\sqrt{\sum_{n} S_{i}\left(\omega_{n}\right) \Delta \omega} .
$$

Table 1 details the irregular waves tested with the peak frequency and significant wave height originally requested as well as those computed here. In addition, further significant wave heights have been calculated for the irregular wave spectra following filtering above $1.5 f_{p}$ (i.e. setting all energy above this cutoff frequency to zero and recomputing significant wave height). The aim of this filtering is to eliminate the effects of energy in higher harmonics, beginning with second harmonic energy principally near to $2 f_{p}$.

Using the procedure above, mean wave spectra for the irregular wave tests with the model present were produced. However, the free surface elevation time histories recorded at different positions in the vicinity of the model clearly represent distinctly different diffraction effects, and so averaging across individual wave spectra at each wave probe position was not performed. This resulted in 52 mean diffracted wave spectra for each incident spectrum (13 wave probe positions for two model headings with two wave probe configurations each).

\subsubsection{Random seas}

For comparison with first-order diffraction theory applied to random seas, the experimental mean-square free surface elevation $\overline{\eta^{2}}$ was calculated for the incident and diffracted wave spectra. Experimental mean-square free surface elevations at a point $(x, y)$ in the diffracted and incident fields were calculated through

$$
\overline{\eta_{d}^{2}}(x, y)=\sum_{n} S_{d}\left(x, y, \omega_{n}\right) \Delta \omega
$$

and

$$
\overline{\eta_{i}^{2}}=\sum_{n} S_{i}\left(\omega_{n}\right) \Delta \omega
$$

where $S_{d}\left(x, y, \omega_{n}\right)$ and $S_{i}\left(\omega_{n}\right)$ are the mean energy density spectra for the diffracted and incident waves, respectively, and $\Delta \omega=2 \pi \Delta f$.

The corresponding theoretical equivalent mean-square free surface elevation was produced using the first-order diffraction theory developed above as

$$
\overline{\eta_{t d}^{2}}(x, y)=\sum_{n}\left|\phi_{n}(x, y)\right|^{2} S_{i}\left(\omega_{n}\right) \Delta \omega,
$$

where $\phi_{n}(x, y)$ is the complex diffraction coefficient (the first-order modification to free surface elevation) for the $n$th spectral component. The incident spectrum $S_{i}\left(\omega_{n}\right)$ 


\begin{tabular}{ll}
\hline Symbol & Description \\
$\eta_{I C}(t)$ & largest incident wave crest from the empty basin tests \\
$\eta_{I T}(t)$ & deepest incident wave trough from the empty basin tests \\
$\eta_{D}(t)$ & diffracted free surface occurring at the same time as $\eta_{I C}(t)$ above \\
$\eta_{D C}(t)$ & largest diffracted free surface (not necessarily identical to $\eta_{D}(t)$ above) \\
$\eta_{D T}(t)$ & deepest diffracted free surface \\
$\eta_{I}(t)$ & incident free surface occurring at the same time as $\eta_{D C}(t)$ above \\
& TABLE 2. Mean focused wave group time histories. \\
\hline
\end{tabular}

is assumed to conform to the mean spectrum for each test as calculated through the method described above.

In addition, to assess the effects of nonlinearity on the experimental results and with the intention of obtaining a closer match between linear theory and nonlinear experiment, further mean-square free surface elevations were calculated for the wave spectra following filtering above $1.5 f_{p}$. This was accomplished by setting all energy above this cutoff frequency to zero and recomputing both experimental and theoretical mean-square free surface elevation using the filtered incident and diffracted spectra.

\subsubsection{Focused wave groups}

Extensive sorting of the crests and troughs was then necessary to provide comparisons with focused wave group theory. As removal of every other data point from the original data could be thought to cause slight deviations in the maxima and minima of troughs and crests, the original data (and not the truncated data described in $\S 3.1 .2)$ were analysed.

The average shape of the five largest crests was computed for both the empty tank tests and the tests with the model present. In order to assess properly the effects of diffraction, six separate mean focused wave group time histories $\eta(t)$ were identified as in table 2.

As the above measured focused wave groups were to be compared with a first order diffraction theory, they were linearized by both filtering the measured spectra and implementing crest-trough comparisons. The spectral filtering was accomplished by computing the FFT of the measured data, setting all frequency components above $1.5 f_{p}$ to zero, and computing the inverse FFT for a new filtered data set. Cresttrough comparison was then implemented for time histories $\eta_{I C}(t)$ and $\eta_{D C}(t)$ using the procedure of Jonathan \& Taylor (1995). Even-order nonlinearity can be removed and a close-to-linear crest time history computed through

$$
\eta_{I C}^{1}(t)=\frac{\eta_{I C}(t)-\eta_{I T}(t)}{2}
$$

and

$$
\eta_{D C}^{1}(t)=\frac{\eta_{D C}(t)-\eta_{D T}(t)}{2},
$$

for incident and diffracted waves, respectively.

The incident focused wave group theory presented above was used to compute a theoretical comparison to the linearized largest incident wave $\eta_{I C}^{1}(t)$ through

$$
\eta_{I C}^{T}(\tau)=\frac{\alpha}{\sigma^{2}} \sum_{n} C_{i n} \operatorname{Re}\left\{\mathrm{e}^{-\mathrm{i} \omega_{n} \tau}\right\},
$$




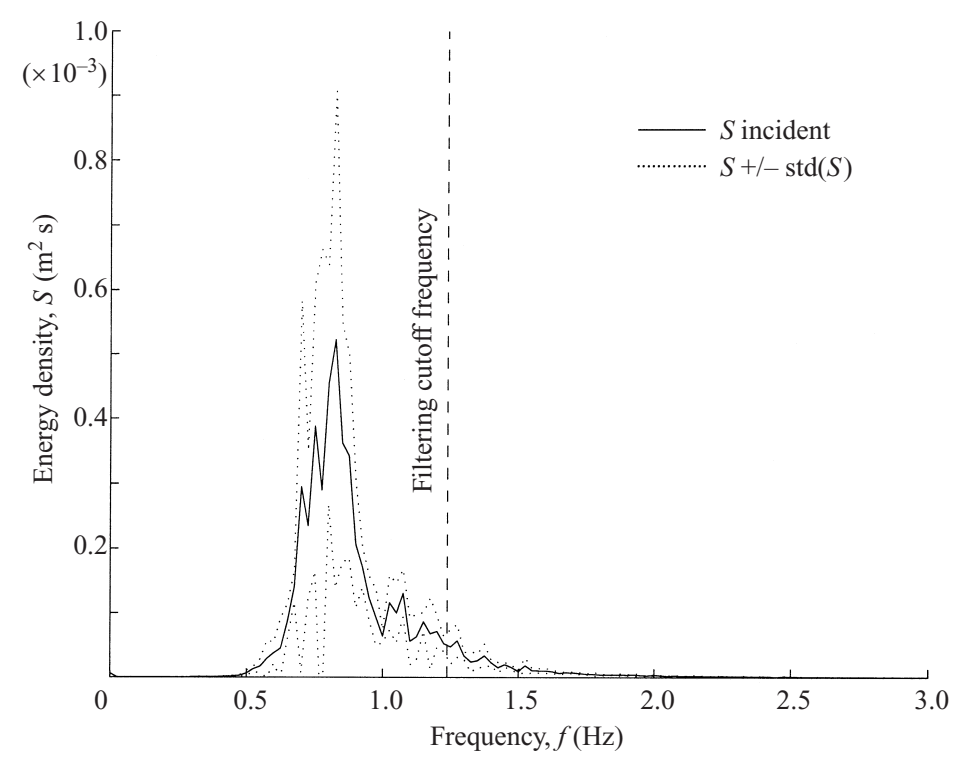

FIGURE 15. Incident wave spectrum 1.

where $C_{i n}=\left(S_{i}\left(\omega_{n}\right) \Delta \omega_{n}\right), \sigma$ is the standard deviation of the incident spectrum, $\alpha$ is the maximum elevation of the incident focused wave group, and $\tau$ is the time interval from the time of occurrence at $\tau=0$.

For comparison to the diffracted largest incident wave $\eta_{D}(t)$ and the linearized largest diffracted wave $\eta_{D C}^{1}(t)$, theoretical diffracted focused wave groups were produced using the first-order diffraction theory and based on the measured diffracted spectrum. For the diffraction of the largest measured incident wave, the amplitude components of this event were treated as deterministic, and the diffracted time history was computed through

$$
\eta_{D}^{T}(x, y, \tau)=\frac{\alpha}{\sigma^{2}} \sum_{n} C_{i n} \operatorname{Re}\left\{\phi_{n}(x, y) \mathrm{e}^{-\mathrm{i}\left(k_{n} x_{0}+\omega_{n} \tau\right)}\right\},
$$

where the position of occurrence of the large crest is $x_{0}$. In contrast, to compute the average shape of the largest waves in the diffracted field, it is necessary to compute the effects of diffraction on the amplitude components of the spectrum. This may be accomplished through

$$
\eta_{D C 1}^{T}(x, y, \tau)=\frac{\alpha}{\sigma^{2}} \sum_{n} C_{i n} \operatorname{Re}\left\{\left|\phi_{n}(x, y)\right|^{2} \mathrm{e}^{-\mathrm{i}\left(k_{n} x_{0}+\omega_{n} \tau\right)}\right\} .
$$

In addition, a subsequent theoretical comparison has been computed from the measured diffracted spectrum at each wave probe location as

$$
\eta_{D C 2}^{T}(x, y, \tau)=\frac{\alpha}{\sigma^{2}} \sum_{n} C_{d n}(x, y) \operatorname{Re}\left\{\mathrm{e}^{-\mathrm{i}\left(k_{n} x_{0}+\omega_{n} \tau\right)}\right\},
$$

where $C_{d n}(x, y)=\left(S_{d}\left(x, y, \omega_{n}\right) \Delta \omega_{n}\right)$ and $S_{d}\left(x, y, \omega_{n}\right)$ is the measured diffracted spectrum as computed from the time history $\eta_{d}(t)$ at the point $(x, y)$. 

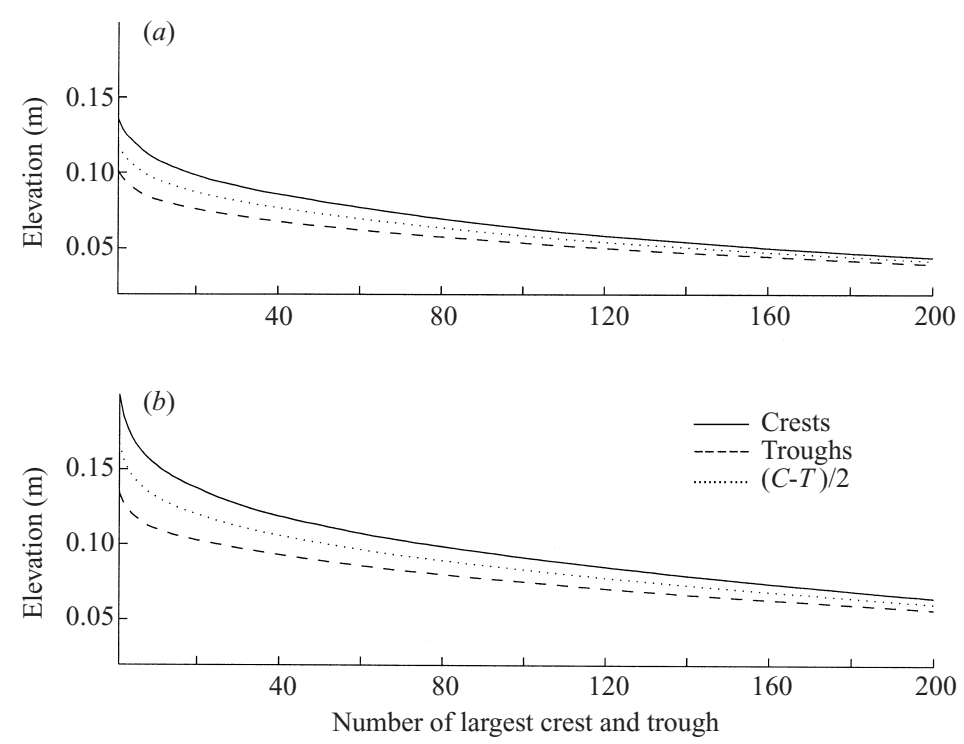

FIGURE 16. Average incident and diffracted large crests and troughs, test 1: $(a)$ mean incident waves from empty tank $\left(f_{p}=0.82499 \mathrm{~Hz}, H_{s}=0.11407 \mathrm{~m}\right) ;(b)$ mean diffracted waves from both headings (all probe positions).

\subsection{Definition of incident wave spectra}

The mean incident wave spectrum produced by the above method was defined to be the incident wave spectrum to be used for theoretical calculations and data nondimensionalization. For the three incident irregular wave conditions, the incident wave spectrum for test 1 is presented in figure 15 as a plot of energy density $S$ (in units of $\mathrm{m}^{2} \mathrm{~s}$ ) versus frequency $f$ (in units of $\mathrm{Hz}$ ), and the $x$-axis scale has been plotted from 0 to $3 \mathrm{~Hz}$. The range plotted demonstrates that the majority of the incident wave energy present during the tests was within the frequency band $0.5 \mathrm{~Hz}<f<1.5 \mathrm{~Hz}$, and that no significant set-up or set-down was present in the incident condition. The solid line plots the mean incident wave spectrum, while the dotted lines plot one standard deviation above and below the mean, as computed from the four separate sections of data identified. Finally, the vertical dashed line indicates the cutoff frequency for filtering at $1.5 f_{p}$ as discussed previously.

\subsection{Definition of incident focused wave groups}

As described above, linearized incident focused wave group crests were computed from the mean free surface elevation time history $\eta(t)$ of the five largest crests and five deepest troughs from a filtered incident spectrum. The distribution of these crests and troughs and their linearized output is demonstrated in figure 16 for test 1 . Not limited to only the five largest crests and deepest troughs, the number of large events plotted has been extended to 200 to present the relationship between the most extreme events and those of more common occurrence with elevation on the order of the significant wave height $H_{s}$. Figure 16(a) demonstrates the maximum elevations from the tests in the empty wave basin, while $(b)$ contains the mean maximum diffracted free surface elevation as computed from an average maximum at all the probe positions with the model in the basin. The solid line plots the maximum elevation of the largest crest $\eta_{I C}$ and $\eta_{D C}$, while the dashed line plots the maximum depth of the corresponding 


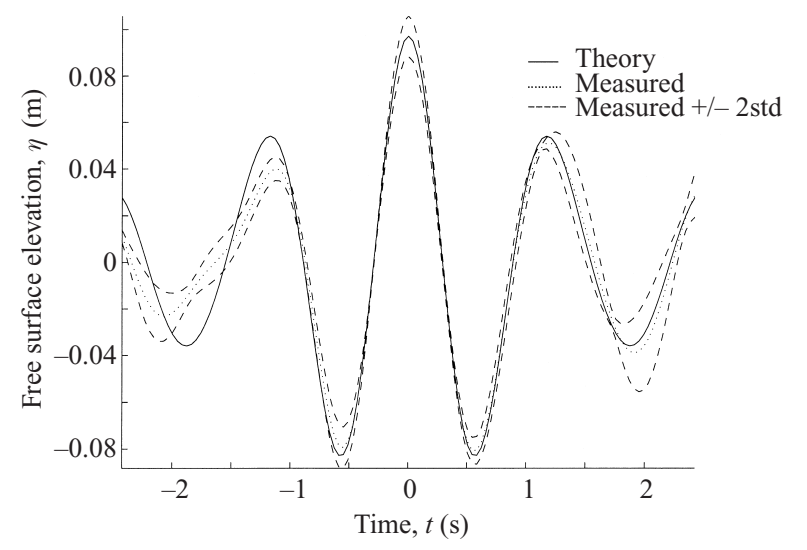

FIGURE 17. Average of five largest incident linearized crests, test 1.

trough $\eta_{I T}$ and $\eta_{D T}$, and the dotted line plots the corresponding linearized result $\eta_{I C}^{1}=\left(\eta_{I C}-\eta_{I T}\right) / 2$ and $\eta_{D C}^{1}=\left(\eta_{D C}-\eta_{D T}\right) / 2$.

From figure 16, it is apparent that the elevation of the $n$th extreme event decreases roughly exponentially, as expected, with increasing $n$. The maximum elevation of the crests is also clearly larger than the depth of the troughs, with linearized crest elevation falling reasonably between the two values. While the averaging of all probe positions renders the diffracted results difficult to interpret, these plots display considerably larger extreme values relative to the incident measurements for both crest and trough, which is indicative of the amplification of large events due to scattering. In addition, while the five largest events vary in maximum elevation by some $15 \%$, they clearly represent extreme elevations relative to the significant wave from each spectrum, with the incident linearized extreme elevation $\eta_{I C}^{1}$ for $n=1$ of the order of $H_{s}$ for all three spectra.

Results of the linearized time series representing incident focused wave groups (in the absence of the model) are plotted and compared to the theoretical focused wave group in figure 17 for test 1 . The dotted line indicates the linearized crest $\eta_{I C}^{1}$ while the solid line plots the theoretical comparison $\eta_{I C}^{T}$ as computed from the incident spectra. The theoretical result has been scaled such that its maximum elevation coincides with that of the experimentally measured incident wave. The dashed lines trace an envelope two standard deviations above and below the mean experimental value $\eta_{I C}^{1}$.

For all three spectra, the theoretical focused wave group remains within this error envelope near to the time of focus $(t=0)$ up to the first crest before or after the focus event $\left(t \approx \pm 1.3 \mathrm{~s} \approx T_{p}\right)$. This result demonstrates the validity of focused wave group theory when applied to the largest events recorded in a random sea. However, it also shows that the applicability of this theory is limited to short time intervals near to the focus event, as the dependence of the free surface on the extreme event decreases with increasing time before or after the event as discussed above.

In addition, it should be noted that the agreement between theory and experiment is significantly better after the focus event than before, again for all three spectra. This may be seen most clearly in figure 17 for test 1 , in which the depth, shape and time of occurrence of the trough at $t \approx 1.8 \mathrm{~s}$ are matched very well by the theory. In contrast, the corresponding trough before the focus event at $t \approx-1.8 \mathrm{~s}$ is poorly reproduced, with the theoretical depth outside the estimated error range 


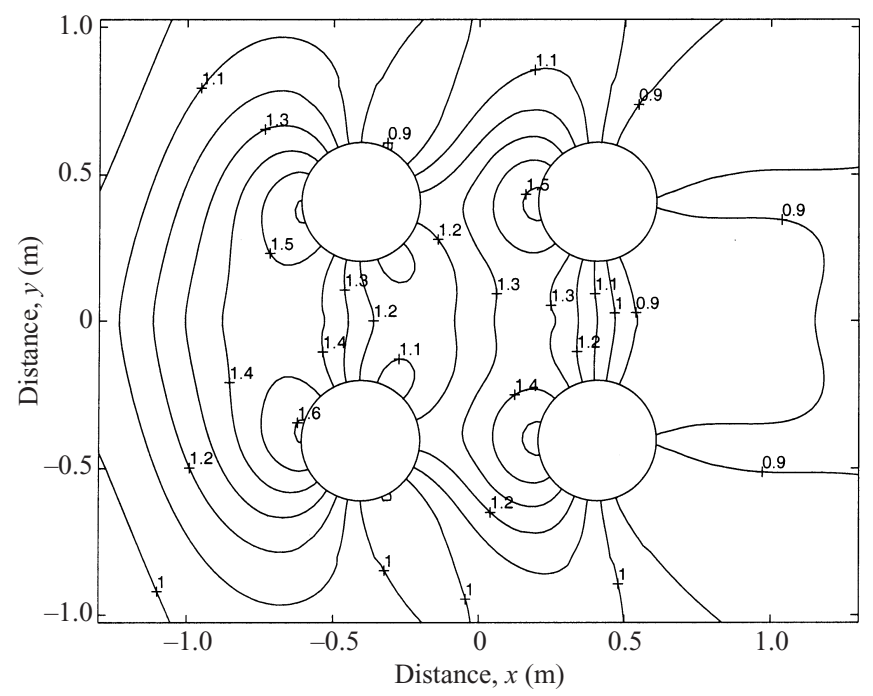

FIGURE 18. Diffracted modification to incident wave spectrum; test 1 , heading $0^{\circ}$.

of two standard deviations and a phase shift of some $0.2 \mathrm{~s}$ evident in the time of occurrence. The consistently better agreement after the focus time for all spectra may be indicative of the nonlinear formation of extreme events. This phenomenon is presumably related to the resonant interactions between frequency components of a very steep focused wave group and has been discussed in more detail by Taylor \& Ohl (1999).

\subsection{Contour plots}

To better understand the general nature of the scattering present during these tests, contour plots of scattering for the three incident wave conditions were produced through the analytical method developed in $\S 2.3$. These contour plots are presented in figure 18 for heading $0^{\circ}$ and figure 19 for heading $45^{\circ}$. Solid circles denote the cylinder positions while key contours are labelled with magnitude in terms of the amplification modifying the incident spectrum at that point (in this case the modification of root-mean-square elevation $\sqrt{\overline{\eta_{d}^{2}(x, y)} / \overline{\eta_{i}^{2}}}$ ). Discussion of these plots is separated according to heading, including some comparison with the equivalent regular wave contour plots from Part 1.

\subsubsection{Heading $0^{\circ}$}

The principal features of the contour plot are significant runup on the front faces of all cylinders as well as broad regions of upwelling in the model centre and upwave of the model. However, a region of somewhat diminished energy $\left(\sqrt{\overline{\eta_{d}^{2}(x, y)} / \overline{\eta_{i}^{2}}}=0.9\right)$ does exist just downwave of the downwave cylinders. In a similar manner to what was observed in the regular wave tests, any modification to the incident wave spectrum decreases significantly with increased distance from the model. This is particularly true for transverse distances, in which very little scattering appears to occur more than two cylinder diameters outboard of the model centre.

Significant first-order runup and upwelling effects are noted in figure 18 for test 1 , for which the incident spectrum is amplified by as much as $50 \%$ and $60 \%$ on the front face of the downwave and upwave cylinders, respectively. In addition, upwelling 


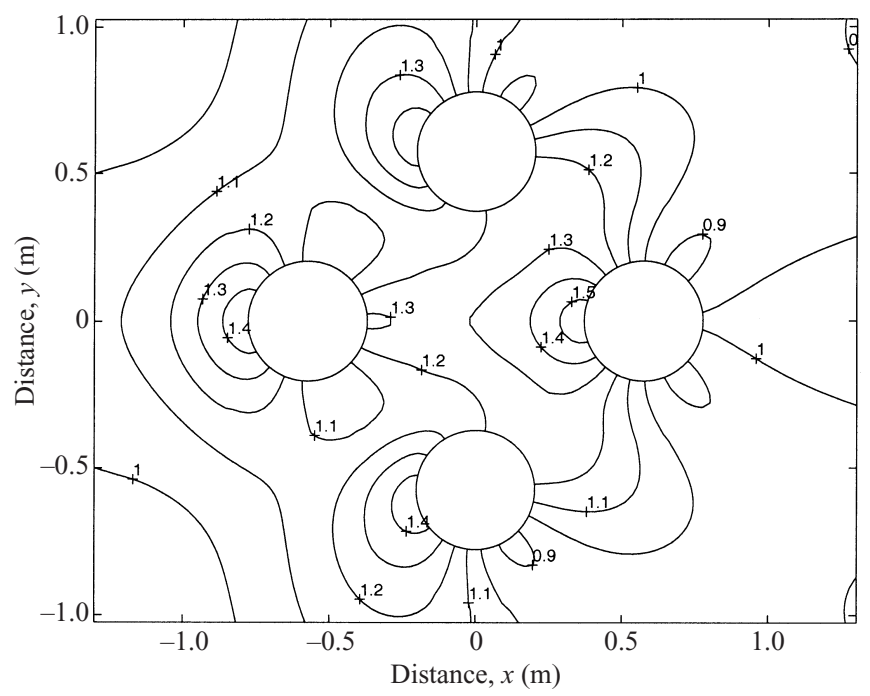

FIGURE 19. Diffracted modification to incident wave spectrum; test 1 , heading $45^{\circ}$.

at the model centre is amplified by between $20 \%$ and $30 \%$ for the three incident spectra.

Figure 18 is very similar to the correponding contour plot for regular wave diffraction at this heading given in Part 1. Both indicate runup at the front face of all four cylinders and regions of upwelling at the model centre and upwave of the model. However, the regular wave contour plots show regions of significant destructive interference and diminished wave amplitude between the upwave cylinders. In addition, the diffracted field clearly has stronger effects at larger distances from the model centre in the regular wave cases. The upwelling upwave of the model is also significantly greater than the runup at the front face of the upwave cylinders in the regular wave case, the reverse of which is obtained here for irregular waves. It is reasonable to suggest that these differences are due to the range of frequencies contributing to the irregular wave contours, which has the effect of smoothing any regions of enhanced or reduced response.

\subsubsection{Heading $45^{\circ}$}

As for the $0^{\circ}$ heading, the $45^{\circ}$ heading contour plot features significant runup on the front faces of all cylinders. A broad region of upwelling exists throughout the model centre, and regions of diminished energy are virtually non-existent, apart from very small regions on the outboard edges of the offset and downwave cylinders in figure 19. Also, almost no modification to the incident spectrum is observed more than two cylinder diameters outboard in the transverse direction, three diameters upwave, or two diameters downwave from the model centre. Runup on the offset and downwave cylinder front faces is at $40 \%$ and $50 \%$ respectively, while centre upwelling is roughly $30 \%$.

Comparing this irregular wave contour plot to the regular wave plots of Part 1, very similar conclusions may be drawn as for the $0^{\circ}$ heading above. While the overall features are very similar, with runup at the front faces of all cylinders and upwelling at the model centre, the more complex features of the regular wave plots appear to be considerably smoothed for the irregular wave case. This is again true at larger distances from the model centre. 

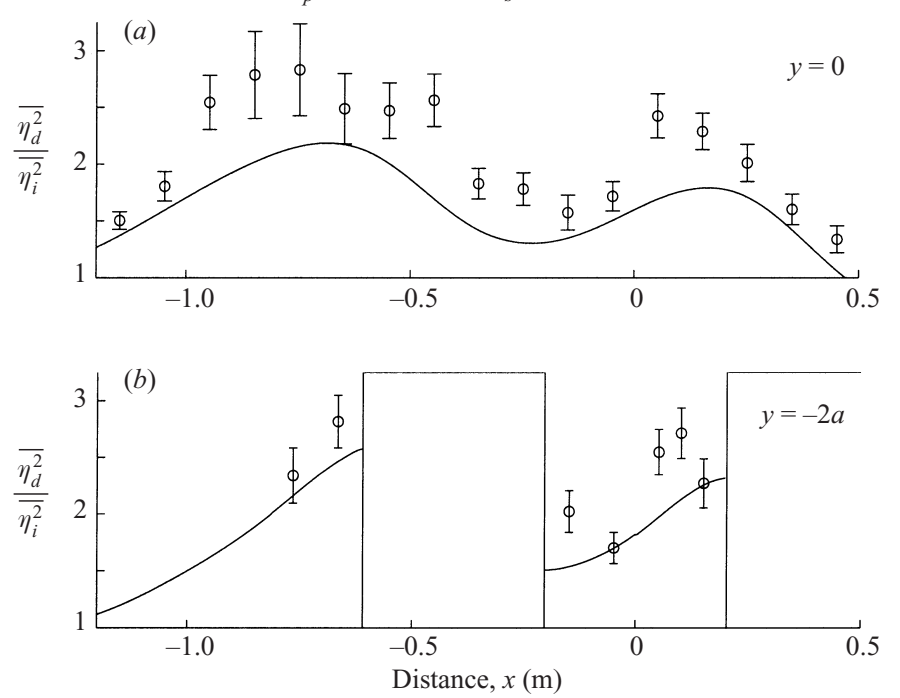

FIGURE 20. Mean square free surface elevation $\overline{\eta^{2}}$ vs. position; test 1 , heading $0^{\circ}$ : circles, experimental results; lines, theory.
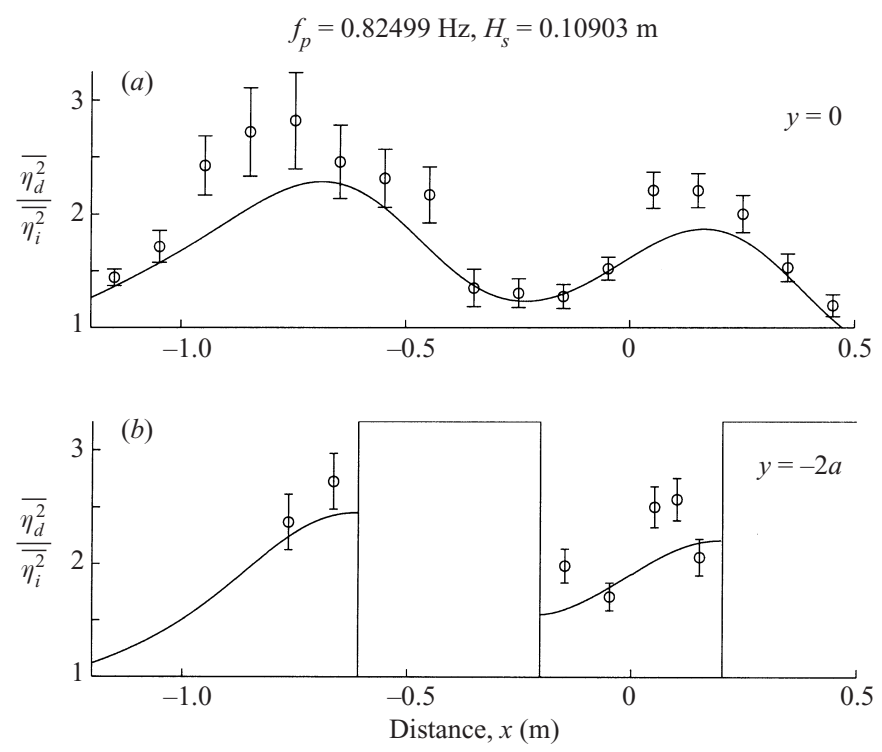

FIGURE 21. Mean square free surface elevation $\overline{\eta^{2}}$ vs. position; test 1 , heading $0^{\circ}$; spectra filtered above $1.5 f_{p}$.

3.5. Mean square free surface elevation $\overline{\eta^{2}}$ and linear theory comparison

Wave probes were placed principally along the centreline of the model $(y=0)$ for both headings. However, for the $0^{\circ}$ heading tests additional probes were placed along a line between the centres of the upwave and downwave cylinders (at $y=-2 a)$, while, for the $45^{\circ}$ heading tests, probes were placed upwave and downwave of the cylinder abeam the tank centre (at $y \approx-2.83 a$ ). Plots of mean-square free surface elevation versus position were made for the three incident irregular waves and the two headings tested. 
$f_{p}=0.82499 \mathrm{~Hz}, H_{s}=0.11407 \mathrm{~m}$
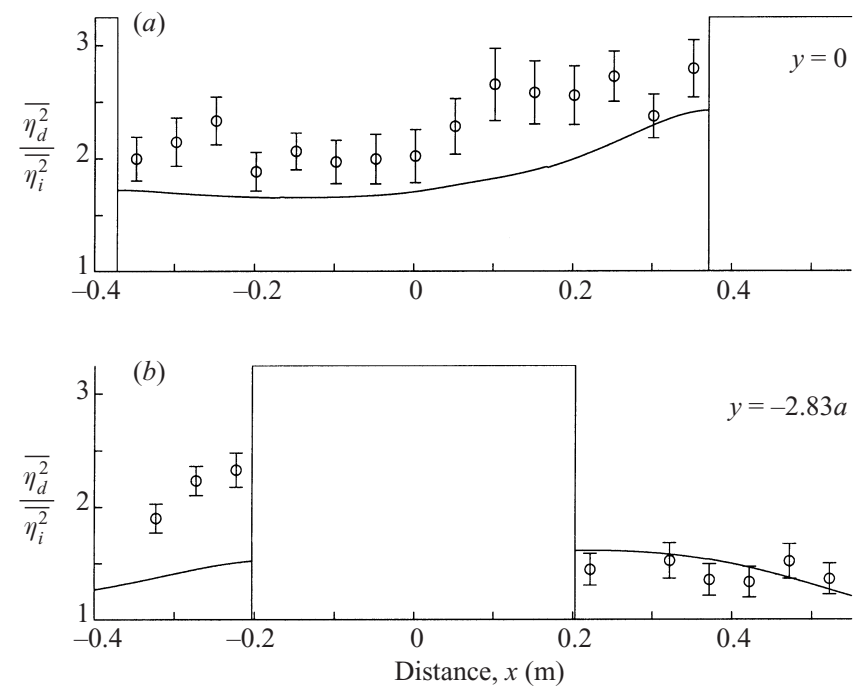

Figure 22. Mean square free surface elevation $\overline{\eta^{2}}$ vs. position; test 1 , heading $45^{\circ}$.

$$
f_{p}=0.82499 \mathrm{~Hz}, H_{s}=0.10903 \mathrm{~m}
$$
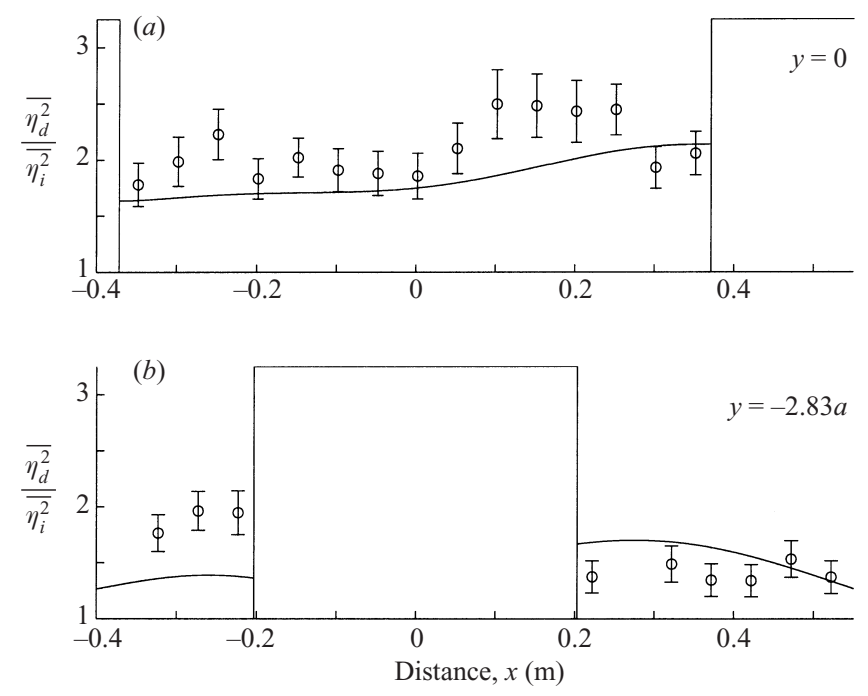

FIGURE 23. Mean square free surface elevation $\overline{\eta^{2}}$ vs. position; test 1 , heading $45^{\circ}$; spectra filtered above $1.5 f_{p}$.

As discussed above, mean-square free surface elevation was calculated for both the complete incident and diffracted spectra as well as for linearized spectra, with all frequency components above $1.5 f_{p}$ removed. Separated into two figures (unfiltered and filtered) for each frequency and heading combination, results for irregular wave case 1 are presented in figures 20 and 21 for heading $0^{\circ}$ and figures 22 and 23 for heading $45^{\circ}$. Plot $(a)$ presents mean-square free surface elevation along the centreline of the model, while plot $(b)$ presents the offset elevation.

Mean-square free surface elevation was non-dimensionalized through division by 
the incident value $\overline{\eta_{i}^{2}}$ of each incident spectrum. Thus, the experimental mean-square free surface elevation $\overline{\eta_{d}^{2}} / \overline{\eta_{i}^{2}}$ has been plotted at the measured points with a circle indicating the mean value and error bars drawn one standard deviation above and below the mean. The theoretical values $\overline{\eta_{t}^{2}} / \overline{\eta_{i}^{2}}$ from linear diffraction theory have been plotted as a continuous solid line.

As in Part 1, regions or point locations of vigorous wave activity due to spectral amplification are referred to as peaks, while regions of diminished wave activity are referred to as troughs. Again, use of these words bears no resemblance to the peaks and troughs of individual waves as measured in the time or spatial domains.

\subsubsection{Heading $0^{\circ}$}

The comparison of experimental results with linear theory is good, particularly along the model centreline. The majority of the global trends present in the theory (solid lines) are followed by the experimental results (circles). However, there is a consistent discrepancy in the elevation, with experimental mean-square free surface elevation up to $25 \%$ higher than the theoretical prediction at some points. This discrepancy is somewhat improved in the filtered spectrum.

For the centreline plots, two large peaks are apparent in both the theoretical and experimental results, one approximately three cylinder radii $(3 a)$ upwave of the model centre and the other approximately one cylinder radius downwave of the model centre, separated by a trough approximately one cylinder radius upwave $(x \approx-a)$.

The only major departure from the theoretical trend is present in the transition from the upwave peak to the trough at $x \approx-2.2 a$, located between the centres of the upwave cylinders. For the unfiltered spectra, the experimental mean-square free surface elevation at this point indicates a further peak followed by a more drastic transition to the trough value. However, this discrepancy is almost completely removed by filtering the spectra, and so this further peak is clearly the result of some nonlinear response near this point, generating energy above the filtering cutoff at $1.5 f_{p}$.

The offset plot demonstrates some consistent differences between the theoretical trends and the experimental results. First-order theory clearly predicts runup at the front faces of both cylinders (as demonstrated in the contour plot of figure 18) with amplification steadily increasing towards the front face of both cylinders. While this is supported for the most part by the two experimental values upwave of the upwave cylinder (showing increased amplification towards the front face), the results between the two cylinders are less in agreement with theory. In addition, the experimental results indicate a peak in runup at the rear face of the downwave cylinder (exceeding the first-order theory again by as much as $25 \%$ ), followed by a trough near the transverse centreline at $x=0$, and a further peak just upwave of the downwave cylinder. This complex behaviour is in many ways similar to that observed in the $0^{\circ}$ heading offset plots of first-order amplitude presented in Part 1. While filtering the spectra marginally improves the agreement between theory and experiment, this discrepancy in the global trend is not affected.

\subsubsection{Heading $45^{\circ}$}

Again, as for the $0^{\circ}$ heading, the similarity between experimental and theoretical results is good, particularly with reference to the global trends. A consistent discrepancy in mean-square amplification of the order of 5\% to $25 \%$ is also present, and this discrepancy is improved somewhat for the filtered spectrum. This is most noticeable in the runup at the front face of the downwave cylinder, which greatly exceeds the theory for the unfiltered plots but shows reasonable agreement after filtering. 


\begin{tabular}{|c|c|c|c|c|c|c|c|}
\hline \multicolumn{4}{|c|}{ Heading $\beta=0^{\circ}$} & \multicolumn{4}{|c|}{ Heading $\beta=45^{\circ}$} \\
\hline $\begin{array}{c}x \\
{[\mathrm{~mm}]}\end{array}$ & & $\begin{array}{c}y \\
{[\mathrm{~mm}]}\end{array}$ & & $\begin{array}{c}x \\
{[\mathrm{~mm}]}\end{array}$ & & $\begin{array}{c}y \\
{[\mathrm{~mm}]}\end{array}$ & \\
\hline $\begin{array}{r}-1150 \\
-350\end{array}$ & $\begin{array}{l}-5.66 a \\
-1.72 a\end{array}$ & $\begin{array}{l}0 \\
0\end{array}$ & $\begin{array}{l}0 \\
0\end{array}$ & -225 & $-1.11 a$ & 757 & $-2.83 a$ \\
\hline
\end{tabular}

TABLE 3. Three wave probe positions for results presented in figures 24 to 29 .

For the centreline plots between the upwave and downwave cylinders, the overall trend in the theoretical results is a region of reasonably constant amplification running from the rear face of the upwave cylinder to just downwave of the model centre $(x \approx 0.15 \mathrm{~m})$. Amplification then increases to the runup location at the front face of the downwave cylinder. However, filtering of the spectra has the effect of somewhat diminishing this increase.

While the experimental results lie near to their theoretical counterparts, there are some notable discrepancies in the complexity of the amplification region. Beginning from the upwave cylinder, the experimental results indicate at least three peaks in amplification at positions varying between $x \approx-1.2 a,-0.7 a, 0.5 a$ and $1.2 a$. Following filtering, the agreement between theoretical and experimental values is somewhat improved, but the global trends persist.

Finally, the offset plot shows a large discrepancy between the theoretical and experimental values upwave of the cylinder, while agreement is much closer downwave. First-order theory predicts somewhat increased amplification approaching the front cylinder face, with somewhat decreasing amplification away from the rear face. While this trend is supported by the experimental results, runup at the front cylinder face is far in excess of the theoretical prediction (by as much as $50 \%$ in figure 22 ). This discrepancy is somewhat improved following filtering. In contrast, the first instance of experimental values falling below the theoretical prediction prior to filtering is evident downwave of the offset cylinder. Following filtering the experimental results are lowered yet further on average. However, this discrepancy is not large and the agreement is still reasonable.

\subsection{Diffracted spectra and focused wave group diffraction}

With diffraction effects unique to each wave probe measurement location and incident frequency, an excessive number of possible comparisons and plots could be presented (with effectively 24 probe positions for two model headings and three incident spectra: 144 possible cases). While all of these cases were analysed, for presentation purposes one incident wave spectrum was selected, from which some significant cases characteristic of all 144 are presented. Based on the contour plot analysis and mean-square free surface elevation results presented above, the incident spectrum 1 (presented in figure 15) was selected as showing the most potential for large modification to the incident spectrum (and therefore a strict test of the first-order focused wave group diffraction theory). For this incident spectrum, three wave probe positions (given in table 3 in terms of cylinder radius and distance in $\mathrm{m}$ ) were selected from the two heading configurations. Plots at these three positions were then produced to compare both spectral and focused wave group computations.

The results of these analyses are presented in figures 24 to 27 for heading $0^{\circ}$ and figures 28 to 29 for heading $45^{\circ}$. The spectra are plotted above the squared diffraction 


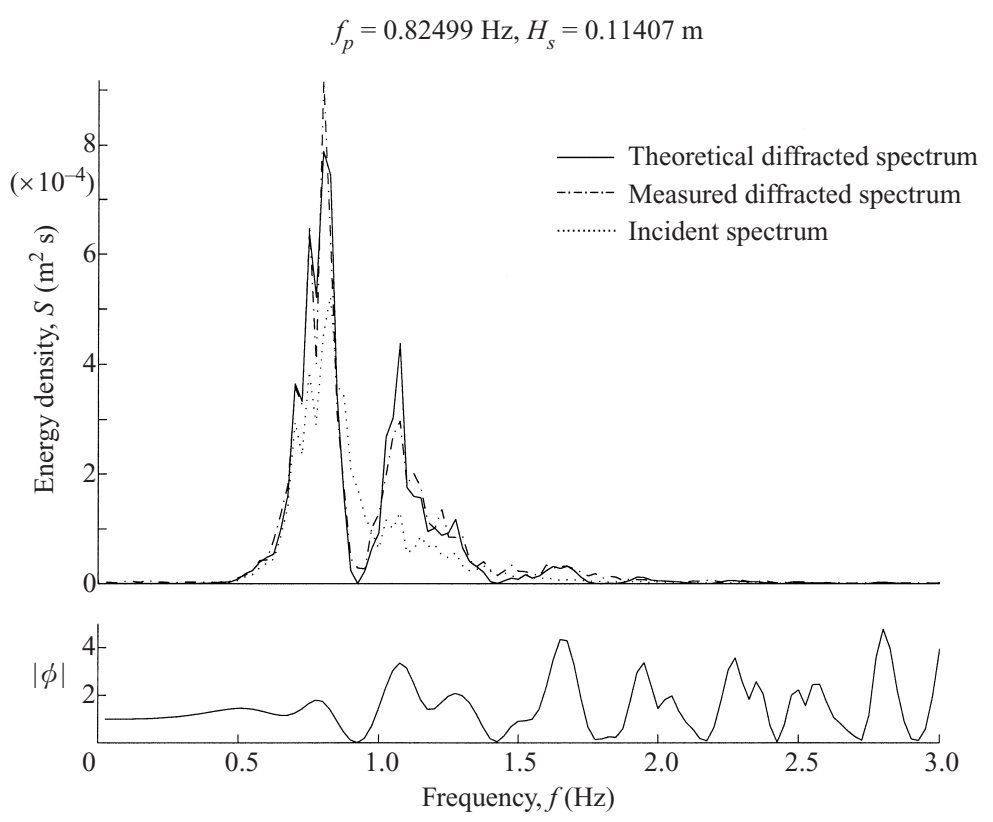

FIGURE 24. Spectral comparison and diffracted modification to incident spectrum at point $(-5.66 a, 0)$; test 1 , heading $0^{\circ}$.

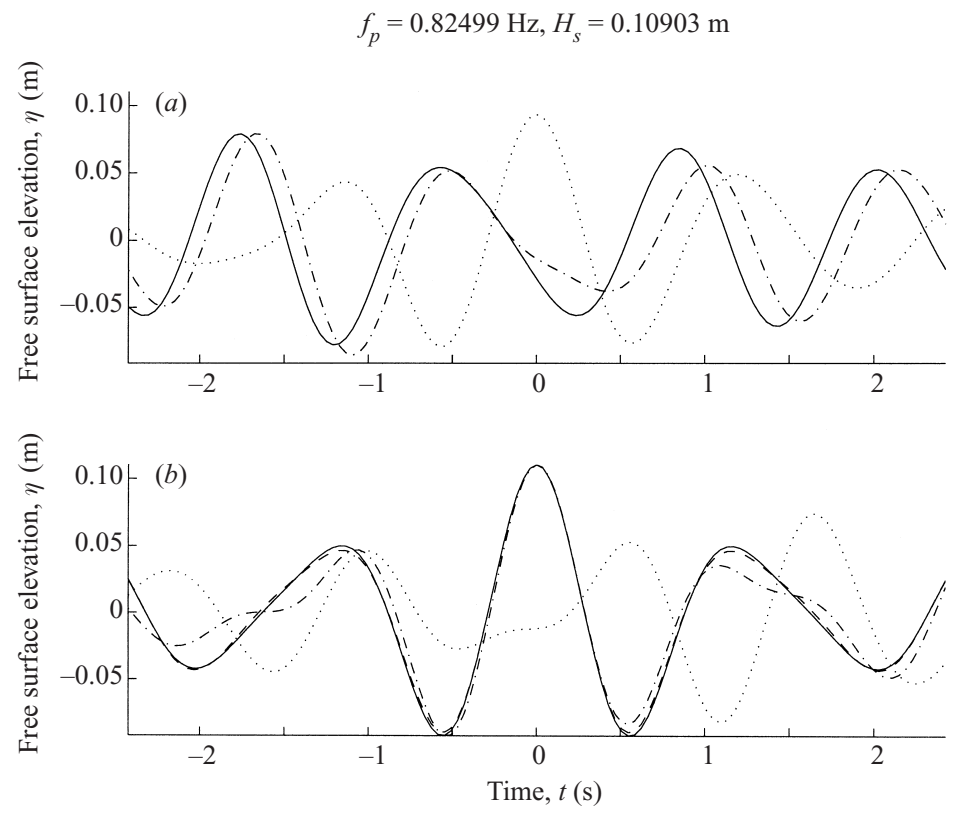

FigURE 25. (a) Incident and $(b)$ diffracted focused wave group comparison at point $(-5.66 a, 0)$; test 1 , heading $0^{\circ}$. For line styles see table 4 .

coefficient $|\phi|^{2}$, which directly modifies the incident wave spectrum $S_{i}$, plotted as the dotted line. The theoretical diffracted spectrum derived from this computation is plotted as the solid line and may be directly compared with the chain dotted line of the measured diffracted spectrum $S_{d}$. 
Wave diffraction. Part 2. Irregular waves

\begin{tabular}{|c|c|c|c|c|}
\hline Symbol & Equation & Description & Part & Line style \\
\hline$\eta_{I C}^{1}(t)$ & (3.7) & $\begin{array}{l}\text { linearized largest incident wave crest } \\
\text { (from empty basin tests) }\end{array}$ & (a) & dotted \\
\hline$\eta_{D}^{T}(t)$ & (3.10) & $\begin{array}{l}\text { theoretical diffracted free surface } \\
\text { occurring at the same time as } \eta_{I C}^{1}(t) \text { above }\end{array}$ & (a) & solid \\
\hline$\eta_{D}(t)$ & $\mathrm{N} / \mathrm{A}$ & $\begin{array}{l}\text { measured diffracted free surface } \\
\text { occurring at the same time as } \eta_{I C}^{1}(t) \text { above }\end{array}$ & (a) & chain dotted \\
\hline$\eta_{D C}^{1}(t)$ & (3.8) & $\begin{array}{l}\text { linearized largest diffracted wave crest } \\
\text { (from model tests in basin) }\end{array}$ & (b) & chain dotted \\
\hline$\eta_{I}(t)$ & $\mathrm{N} / \mathrm{A}$ & $\begin{array}{l}\text { measured incident free surface occurring } \\
\text { at the same time as } \eta_{D C}^{1}(t) \text { above }\end{array}$ & (b) & dotted \\
\hline$\eta_{D C 1}^{T}(t)$ & (3.11) & $\begin{array}{l}\text { first theoretical largest diffracted } \\
\text { wave crest }\end{array}$ & (b) & solid \\
\hline$\eta_{D C 2}^{T}(t)$ & (3.11) & $\begin{array}{l}\text { second theoretical largest diffracted } \\
\text { wave crest }\end{array}$ & (b) & dashed \\
\hline
\end{tabular}

TABLE 4. Key to parts (a) and (b) of focused wave group figures 25, 27 and 29.

As the focused wave group comparisons in figures 25, 27 and 29 are somewhat more complex, table 4 presents a key to these figures. Part $(a)$ displays results for the diffraction of an incident focused wave group (i.e. the diffracted history occurring at the same time as an extreme incident event at $t=0$ ). Part $(b)$ details the largest diffracted event at $t=0$ and the incident event measured at that time. In general, solid and dashed lines indicate theoretical diffracted results, while chain dotted lines represent the measured diffracted results and dotted lines indicate the measured incident wave.

In part $(a)$ of the focused wave group plots, the solid line is the theoretical diffracted free surface elevation time history $\eta_{D}^{T}$, as computed through (3.10), associated with an extreme incident event $\eta_{I C}^{1}$ (the dotted line) occurring at $t=0$ and linearized through crest-trough subtraction. No dashed line is present in part $(a)$, and the chain dotted line represents $\eta_{D}$, the diffracted time history measured at the time corresponding to the incident extreme event.

In part $(b)$, the chain dotted line is the largest measured diffracted free surface elevation time history $\eta_{D C}^{1}$ at $t=0$ produced by an arbitrary incident wave condition $\eta_{I}$ (the dotted line) and linearized through crest-trough subtraction. As a theoretical comparison, the solid line is the largest theoretical diffracted free surface elevation time history $\eta_{D C 1}^{T}$, as computed through (3.11). In contrast, the dashed line in $(b)$ is the theoretical diffracted time history $\eta_{D C 2}^{T}$ as computed from the measured diffracted spectrum through (3.12). Due to the similarity of the measured diffracted spectra and the theoretically computed spectra, these solid and dashed lines overlap significantly and obscure one another in the majority of the plots. It should also be noted that these two lines are symmetrical about $t=0$, while the experimental results (represented by the chain dotted line) are clearly not. In addition, all of these theoretical diffracted free surface time history calculations have been scaled such that their maximum values coincide with the corresponding maximum measured values.

\subsubsection{Heading $0^{\circ}$ : diffracted spectra}

The spectral comparison plots demonstrate that the linear diffraction theory for random seas developed above is extremely effective at reproducing the diffraction effects measured in the experiments. 


$$
f_{p}=0.82499 \mathrm{~Hz}, H_{s}=0.11407 \mathrm{~m}
$$

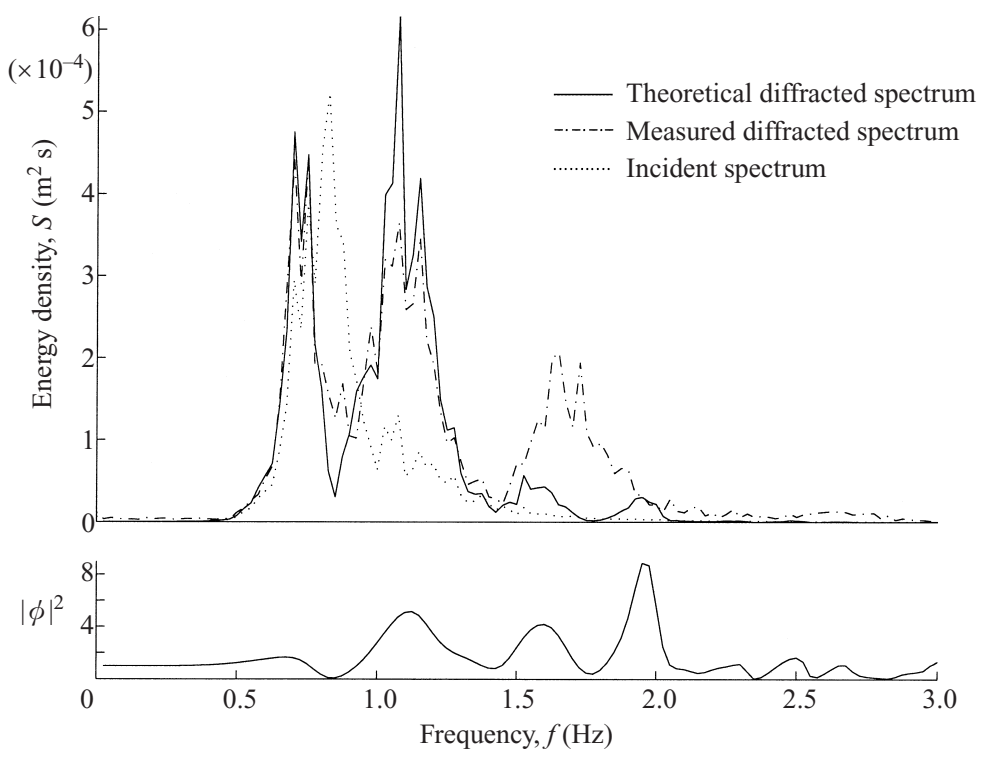

FIGURE 26. Spectral comparison and diffracted modification to incident spectrum at point $(-1.72 a, 0)$; test 1 , heading $0^{\circ}$.

$$
f_{p}=0.82499 \mathrm{~Hz}, H_{s}=0.10903 \mathrm{~m}
$$
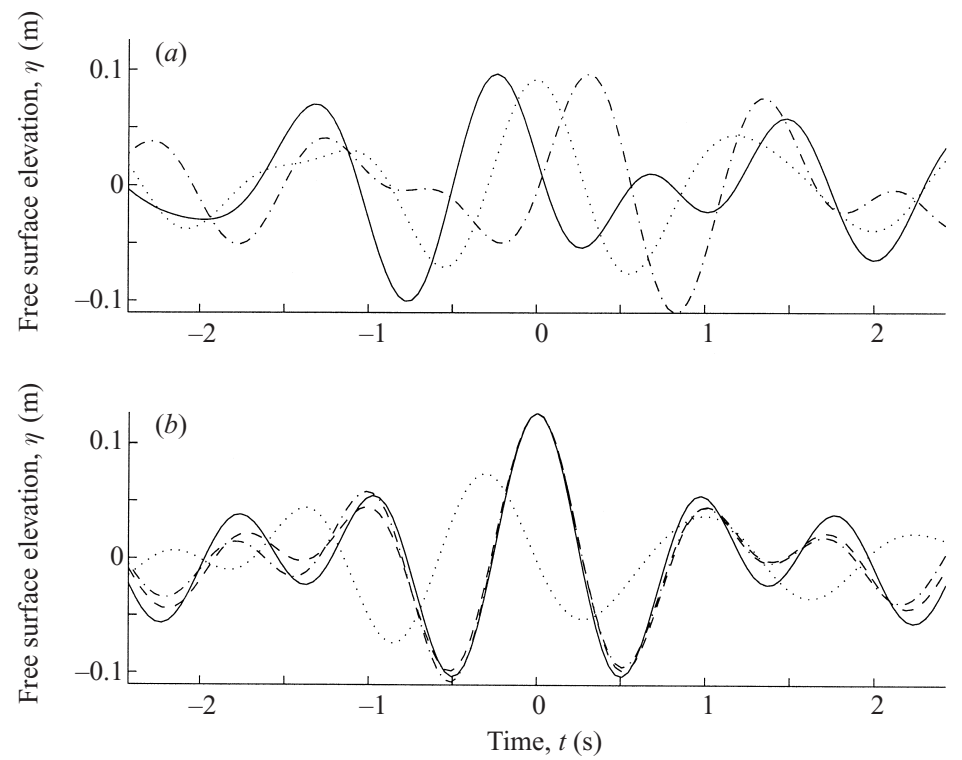

FIGURE 27. (a) Incident and $(b)$ diffracted focused wave group comparison at point $(-1.72 a, 0)$; test 1 , heading $0^{\circ}$. For line styles see table 4 .

Comparison of the plots of squared diffraction coefficient $|\phi|^{2}$ versus frequency $f$ demonstrate the variability of diffraction effects at different points in the vicinity of the model. For example, figure 24 at $(-5.66 a, 0)$ exhibits rapidly varying values, with multiple large peaks of the order of $|\phi|^{2}=4$ separated by zeros at frequencies for 


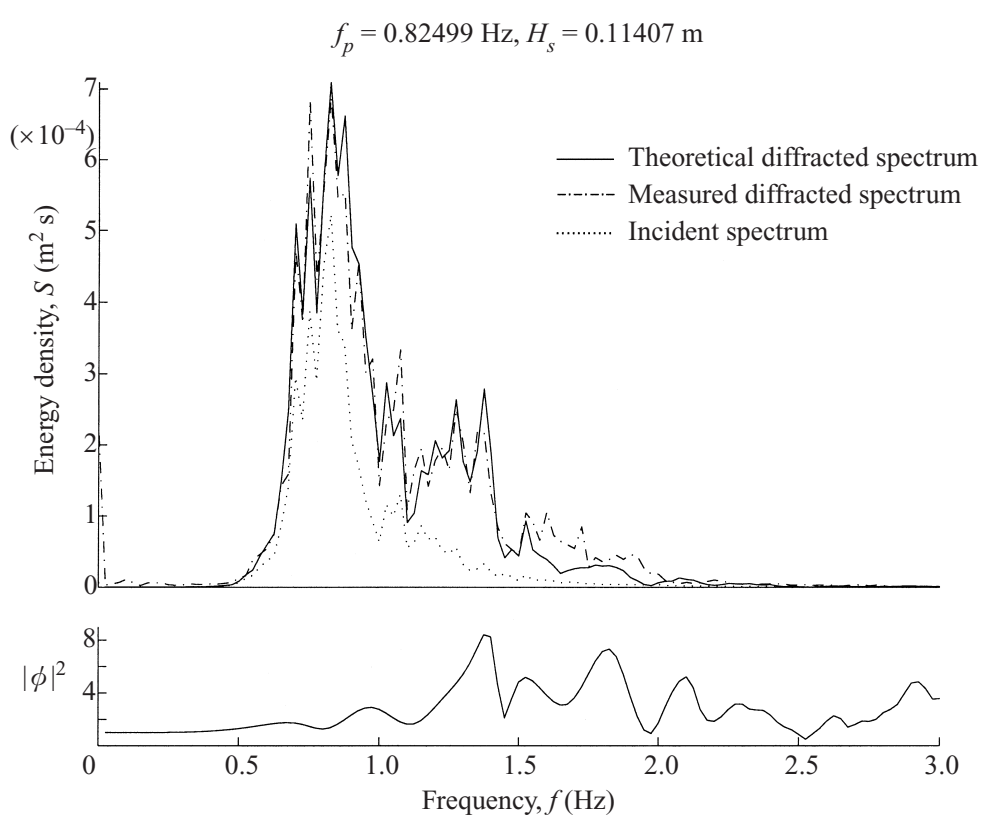

FIGURE 28. Spectral comparison and diffracted modification to incident spectrum at point $(-1.11 a,-2.83 a)$; test 1 , heading $45^{\circ}$.
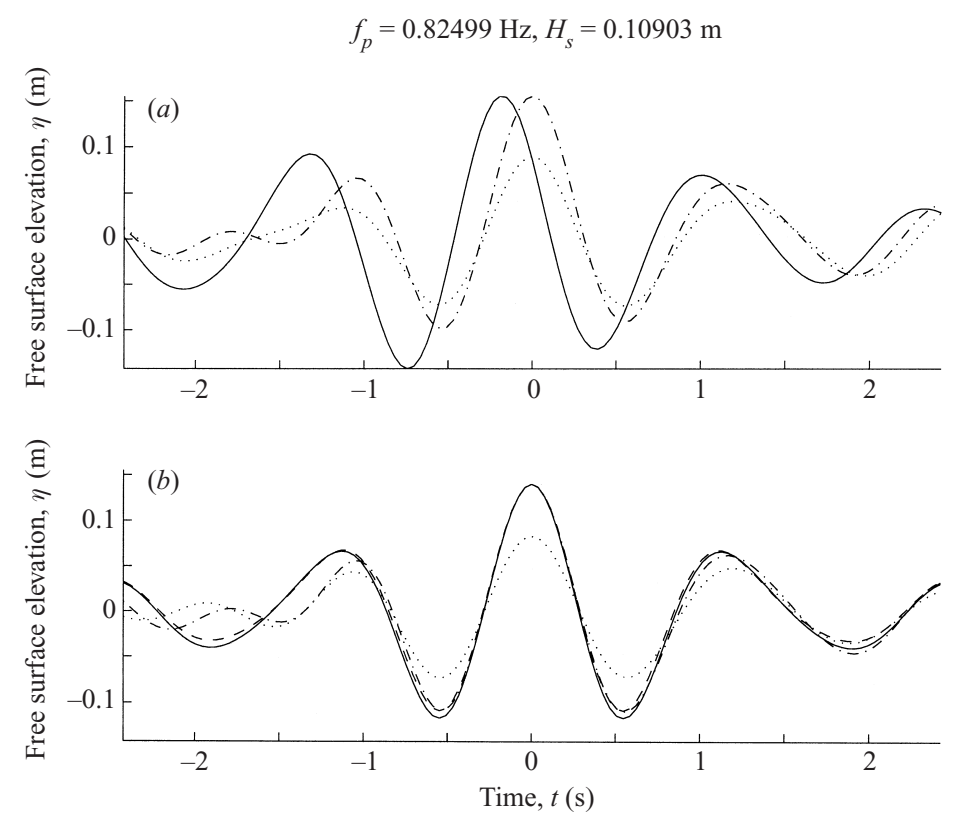

FiguRE 29. (a) Incident and (b) diffracted focused wave group comparison at point $(-1.11 a,-2.83 a)$; test 1 , heading $45^{\circ}$. For line styles see table 4 .

which the incident and scattered waves clearly cancel one another at this point. In contrast, figure 26 at $(-1.72 a, 0)$ exhibits far less variation, particularly for frequencies above $f=2.5 \mathrm{~Hz}$, where the diffraction coefficient again approaches zero.

These differences in response are equally apparent in the spectral comparison plots, 
which also demonstrate the effectiveness with which first-order diffraction theory approximates the fully nonlinear spectral diffraction. For the majority of the plots, the solid lines of the theoretical predictions very nearly approximate the chain dotted lines of the measured spectra. The appearance of zeros in the plots of squared diffraction coefficient $|\phi|^{2}$ is also verified in these plots, such that the $|\phi|^{2}=0$ value at $f \approx 0.95 \mathrm{~Hz}$ in figure 24 corresponds to an exceptionally low value for the measured diffracted spectrum.

However, some notable differences are apparent, particularly where significant influence of nonlinearity may be expected. This is most extreme in figure 26 at $(-1.72 a, 0)$, which represents a wave probe location along the centreline in between the upwave cylinders. In this case, a relatively large band of energy, with peak value approximately $40 \%$ of the incident spectrum peak, is present in the measured diffracted spectrum within the frequency band $1.5 \mathrm{~Hz}<f<2 \mathrm{~Hz}$, which may be taken to lie near to a second-order response $\left(2 f_{p}\right)$ for the incident spectrum. It may also be suggested that the presence of this second-order nonlinearity contributes to the discrepancies found between the theoretical and measured diffracted spectra in the trough region at $f \approx 0.9 \mathrm{~Hz}$ and the second peak at $f \approx 1.1 \mathrm{~Hz}$. It is worth noting that, for both of these nonlinear measured peaks, the corresponding plots of squared diffraction coefficient $|\phi|^{2}$ show near zero values. This indicates that these nonlinear peaks are clearly not the result of linear diffraction and that the discrepancy between measured and theoretical diffracted spectra at these frequencies may not be attributed to improperly computed incident spectra.

Overall, these discrepancies between measured and theoretical diffracted spectra are the source of the consistent differences observable in the plots of mean-square free surface elevation $\overline{\eta^{2}}$, computed from the area under these spectra. Comparing the positions chosen here with the corresponding $\overline{\eta^{2}}$ calculations in figure 20 , those points exhibiting considerable higher-order behaviour also yield significant discrepancies in mean-square free surface elevation. However, following filtering of the spectra above $1.5 f_{p}$ and removal of the majority of these nonlinear effects, the agreement is much better, as demonstrated in figure 21 .

\subsubsection{Heading $0^{\circ}$ : diffracted focused wave groups}

While some discrepancies are present for the diffracted incident extreme waves (the upper focused wave group subplots), the largest diffracted free surface elevation time histories in the lower subplots are very well approximated by the theoretical results.

It is clear from figure $25(a)$ that, due to the phase shifting of scattered waves, an incident focused wave group crest $\eta_{I C}^{1}$ occurring at time $t=0$ does not necessarily generate an equivalent diffracted large crest at $t=0$. This is most apparent along the model centreline, such that in figure 25 at $(-5.66 a, 0)$ the measured diffracted time history $\eta_{D}$ in the vicinity of the incident extreme event yields no crests even as high as the initial incident event itself.

These measured diffracted free surface time histories $\eta_{D}$ are matched by the theoretical equivalents $\eta_{D}^{T}$ with a varying degree of accuracy. The error in this comparison is heavily dependent on the point chosen, with a reasonable match obtained in figure 25 in terms of both the crest and trough elevations and phase relationships. This is in stark contrast with figure 27 , for which the phase relationships are so badly predicted that the mirror image of the theoretical result appears to give a closer fit to the measured data. Again, this may be attributed to nonlinearity producing the discrepancy between the measured and theoretical diffracted spectra at $f \approx 0.85 \mathrm{~Hz}$ (the previously mentioned higher-order nonlinearities in the band $1.5 \mathrm{~Hz}<f<2 \mathrm{~Hz}$ 
for figure 26 would not be expected to influence the focused wave group result due to filtering above $\left.1.5 f_{p}\right)$.

In figures $25(b)$ and $27(b)$ the two theoretically computed extreme diffracted events $\left(\eta_{D C 1}^{T}\right.$ and $\left.\eta_{D C 2}^{T}\right)$ virtually overlap one another, as expected due to the close agreement between the spectra. In addition, the agreement with the measured linearized extreme diffracted events $\eta_{D C}^{1}$ is extremely good for most of the plots, particularly within the limits of $T_{p}<t<T_{p}$. This agreement carries further for some cases, such as figure 27 at $(-1.72 a, 0)$, in which the complex trend in the time history is very well reproduced to $t \approx \pm 2 \mathrm{~s}$.

The incident free surface time histories $\eta_{I}$ confirm that, due to the phasing of scattered waves from the model, these extreme diffracted events do not necessarily correspond to extreme incident events. This is particularly clear in figure 25 at $(-5.66 a, 0)$, in which the incident time history bears no indication of an extreme event in the vicinity of the extreme diffracted event.

\subsubsection{Heading $45^{\circ}$ : diffracted spectra}

Again, the comparisons of diffracted spectra demonstrate that the linear diffraction theory for random seas is extremely effective at matching the measured diffracted spectra.

The squared diffraction coefficient $|\phi|^{2}$ plots also exhibit substantial differences at each of the four points selected. The most extreme variation is observed in figure 28 at $(-1.11 a,-2.83 a)$, which represents a point very near to the runup at the front face of the offset cylinder. This plot exhibits multiple peaks on the order of $|\phi|^{2}=8$ and rapid variation with frequency. More similarities do not appear in comparisons between plots along the centreline versus those in the vicinity of the offset cylinder (not presented here).

Some notable discrepancies are again present between the measured and theoretically computed spectra, although in this case they are not so easily attributable to higher-order phenomena. A distinct difference may be noted in figures 24, 26 and 28 , for which a low, broad peak may be observed in the measured spectra within a frequency band of approximately $1.5 \mathrm{~Hz}<f<2 \mathrm{~Hz}$. Again, this measured peak corresponds to a relative peak in the squared diffraction coefficient $|\phi|^{2}$. This may then indicate that some of this energy is due to first-order diffraction and is somehow not well reproduced here due to possible errors in the computed incident spectrum. However, it should also be noted that this frequency band corresponds to that of the higher-order energy observed in figure 26 , and could be explained in similar terms as the presence of some second-order response at twice the peak frequency.

\subsubsection{Heading $45^{\circ}$ : diffracted focused wave groups}

As before for the $0^{\circ}$ heading, some discrepancies may be noted in part $(a)$ for diffracted incident extreme waves, while part $(b)$ indicates very good agreement between theory and experiment for the largest diffracted free surface elevations.

At the $45^{\circ}$ heading phase, shifts for the scattered incident extreme events are less clear. This is most evident in figure 29 at $(-1.11 a,-2.83 a)$. In this figure, the time of occurrence of the maximum elevation in the measured diffracted time history $\eta_{D}$ is very nearly $t=0$, the focus time of the incident extreme event $\eta_{I C}^{1}$.

The theoretical diffracted free surface time history $\eta_{D}^{T}$ matches the measured values $\eta_{D}$ with varying success. Figure 29 exhibits less than perfect agreement, such that the phase shift predicted by the theory is virtually absent in the measured diffracted time histories and the trough depth occurring before and after the maximum elevation 
are also poorly predicted. This may possibly be attributed to the predominance of higher frequency energy in this case, which, from figure 28 , contains significant energy above $1.5 f_{p}$. This is in addition to the broad band of nonlinear energy identified in the range $1.5 \mathrm{~Hz}<f<2 \mathrm{~Hz}$. While the components above this frequency have been removed for the focused wave groups presented, it is possible that they have had some influence on the phasing and amplitude of other components at the spectral peak.

In part (b), the two theoretically computed extreme diffracted events $\left(\eta_{D C 1}^{T}\right.$ and $\left.\eta_{D C 2}^{T}\right)$ again produce very similar results due to the close agreement between the spectra. The agreement of these theoretical predictions with the experimentally measured $\eta_{D C}^{1}$ also resembles closely that of the $0^{\circ}$ heading, with exceptional accuracy limited to within the range $T_{p}<t<T_{p}$. As for the incident extreme event presented earlier in figure 17, the agreement is somewhat better after the focus time as opposed to before. This is most apparent in figure 29 at $(-1.11 a,-2.83 a)$, in which a more complex feature of the measured time history $\eta_{D C}^{1}$ at $t \approx-1.8 \mathrm{~s}$ is not reproduced by the theoretical result, while the match after the extreme event $(t>0)$ is extremely close.

In contrast to the $0^{\circ}$ heading, the incident free surface time history $\eta_{I}$ producing the extreme diffracted event is more similar in appearance to an extreme incident crest focusing at $t=0$.

\section{Discussion}

\subsection{Extension of linear diffraction}

Multiple cylinder linear diffraction theory (Linton \& Evans 1990) has been extended to incident focused wave groups through the method of Eatock Taylor \& Wu (1997), who computed the diffraction of focused wave groups from a single cylinder. Through the generalization of first-order focused wave group diffraction theory, a simplified expression has been derived for the maximum free surface elevation $\eta_{m}$ at any point due to a given incident focused wave group. Due to the phasing of the scattered waves from the cylinders, this $\eta_{m}$ is not necessarily achieved by an incident extreme wave crest but may be realized by an incident wave group which is somewhat out of focus. This method is applicable to a deterministic incident focused wave group chosen to correspond to a given incident wave spectrum. The amplitude components of this focused wave group are assumed to be fixed, and the effect of scattering from the cylinder array is computed through linear diffraction.

Returning to the concept of a random sea, the method of Eatock Taylor \& Sincock (1989) has been applied to compute the effects of diffraction on the incident spectral components. This differs from the above result in that the energy in each spectral component is treated as variable, and a modification to this energy is computed through first-order diffraction theory. This method may then be used to calculate statistics $\left(\eta_{d} / \eta_{i}\right)$ for extreme surface responses consistent with an incident random wave field of known spectrum at a given point in the vicinity of a structure; this is also the local enhancement of root-mean-square free surface elevation and significant wave height. While this spectral method produces results similar to those for the diffraction of focused wave groups, significant differences do exist as the two methods differ in their treatment of extreme events.

In summary, two new methods for determining extreme design events have been presented. The first incorporates the effects of linear diffraction on a given incident focused wave group with fixed amplitude components as determined by the incident wave spectrum. In contrast, the second computes a diffraction modification to the incident wave spectrum itself, from which statistics of surface elevation may then be computed. 
4.2. Linear diffraction theory for random seas and focused wave groups

For both headings, the overall agreement between theory and experiment for diffracted spectra and spectral statistics was found to be excellent. Where discrepancies did exist, these could in most cases be attributed to the presence of energy at higher frequencies, which is discussed further below.

Again, first-order theory for focused wave groups has been shown to be effective at reproducing the time histories of extreme events in the vicinity of a model. However, it must be emphasized that the comparisons made here are for filtered spectra, with all components above $1.5 f_{p}$ removed, and linearized extreme events (applying the crest-trough subtraction method of Jonathan \& Taylor 1995).

\subsection{Focused wave group time history agreement for $t>0$}

The theoretical and experimental time histories computed for both incident and diffracted extreme events showed better agreement after the focus time at $t=0$. In the theoretical approach, the computed first-order focused wave groups were symmetrical about the focus time $t=0$ for both the diffracted and incident waves. However, the majority of the measured extreme events were not symmetrical, with some notable differences in the time history before and after the focus time by approximately $t= \pm 1.5 T_{p}$, depending on the case presented. These differences tended towards better agreement with the theoretical computations for times after the focus (i.e. $t>0$ ).

This consistently better agreement after the focus time may be related to nonlinear interactions present during the formation of extreme events. Due to resonant interactions between frequency components of the focused wave group, the group may come into focus and reach an extreme value earlier than is predicted by linear theory, particularly for a reasonably steep extreme event. Linear diffraction theory may then be expected to produce more accurate temporal results after the event, when the point of extreme elevation has passed the point of observation. However, this resonant interaction between frequency components of focused wave groups is discussed in more detail by Taylor \& Ohl (1999).

\subsection{Nonlinearity in diffracted wave spectra}

While nonlinearity in the diffracted spectra has been observed at several wave probe locations for both the $0^{\circ}$ and $45^{\circ}$ headings, a point of particular note was the highfrequency peak in the range $1.5 \mathrm{~Hz}<f<2 \mathrm{~Hz}$ (i.e. of the order $2 f_{p}$ ) observed during the $0^{\circ}$ heading tests at $(-1.72 a, 0)$, which was not predicted by linear theory. In terms of the cylinder radius $a$ and cylinder spacing $2 a$, this measurement position represents a point along the longitudinal model centreline and between the upwave cylinders (just downwave of a line joining the centres of the upwave cylinders at $x=-2 a$ ). As shown in figure 7 from Part 1, which plots second-order amplitude versus position (part $(a)$, centre) for the regular wave frequency nearest to the peak frequency $(f=0.8000 \mathrm{~Hz})$, measurements at this point indicate significant second-order response.

\section{Conclusions}

The primary results of the analyses presented here are as follows:

(a) First-order diffraction theory has been extended to the case of focused wave groups and random seas incident on an array of bottom-mounted circular cylinders.

(b) This linear diffraction theory for random seas has been shown to be an effective method for simulating the shapes of measured spectra. 
(c) Statistics computed from these theoretical diffracted wave spectra have been shown to reproduce global trends in the experimental data. Consistent discrepancies between the theoretical and experimental results may be attributed to nonlinear interactions.

(d) Linear diffraction theory for focused wave groups (also developed herein) has been demonstrated to be an effective model for linearized extreme events, produced through crest-trough subtraction.

(e) The phase shifting of incident wave spectral components has been found to significantly modify the diffracted focused wave groups in the vicinity of the cylinder array.

$(f)$ For both incident and diffracted focused wave groups, the most extreme events recorded in the experimental data have shown improved agreement with theoretical predictions after the focus time $(t=0)$.

$(g)$ High-frequency energy components related to nonlinear interactions have been observed in the measured diffracted spectra.

(h) This nonlinearity has been shown to be particularly dominant at locations between the upwave cylinders for the $0^{\circ}$ heading tests. This is in agreement with results from the regular wave tests presented in Part 1.

The authors acknowledge the support of HR Wallingford and Dr Roger Wilkinson, the former Offshore Group Manager at HR, for the time in the Wallingford offshore wave basin and the construction of the model. The first author was supported through a British Marshall Scholarship from the Marshall Commemoration Commission. The authors are grateful for the constructive comments of the referees.

\section{REFERENCES}

Dean, R. G. \& Dalrymple, R. A. 1991 Water Wave Mechanics for Engineers and Scientists, Vol. 2. World Scientific.

Eatock TAYlOR, R. \& Sincock, P. 1989 Wave upwelling effects in TLP and semisubmersible structures. Ocean Engng 16, 281-306.

EAtock TAYLOR, R. \& Wu, G. X. 1997 Interaction of steep waves with offshore structures. Phil. Trans. R. Soc. Lond. A 355, 593-605.

Jonathan, P. \& TaYloR, P. H. 1995 Irregular, nonlinear waves in a spread sea. ASME Trans. J. Offshore Mech. Arctic Engng 119, 37-41.

LindGREN, G. 1980 Extreme values and crossings for the chi-squared and other functions of multi-dimensional Gaussian processes. Adv. Appl. Prob. 12, 746.

Linton, C. M. \& Evans, D. V. 1990 The interaction of waves with arrays of vertical circular cylinders. J. Fluid Mech. 215, 549-569.

OHL, C. O. G. 1999 Free surface disturbances and nonlinear runup around offshore structures. DPhil thesis, University of Oxford.

Ohl, C. O. G., Eatock Taylor, R., Taylor, P. H. \& Borthwick, A. G. L. 2001 Diffraction by a cylinder array. Part I: regular waves $J$. Fluid Mech. 442, 1-32.

TAYloR, P. H. \& OHL, C. O. G. 1999 Focussed wave groups II: global nonlinearity and resonant wave-wave interactions. 18th Intl Conf. Offshore Mechanics and Arctic Engng. OMAE99/S\&R6462. ASME.

Tromans, P. S., Anaturk, A. R. \& Hagemeijer, P. 1991 A new model for the kinematics of large ocean waves: Application as a design wave. Proc. 1st Intl Offshore and Polar Engineering Conf., Edinburgh, vol. 3, pp. 64-71. 